\title{
Lens-Antenna Coupled Superconducting Hot-Electron Bolometers for Terahertz Heterodyne Detection and Imaging
}

\author{
Lei Liu \\ Department of Electrical Engineering, University of Notre Dame, \\ Notre Dame, IN, \\ USA
}

\section{Introduction}

Both astronomic observation and atmospheric remote sensing in the terahertz $(\mathrm{THz})$ frequency range (0.1-10 THz) have driven the demand for highly-sensitive mixers and receivers [1-4]. Interstellar molecule spectrum information obtained through those $\mathrm{THz}$ receivers provides the basic clues to understand the formation of the universe. Also, the observation and monitoring of the earth's own atmosphere may allow strategies to be developed to address issues such as global warming or ozone depletion [5, 6]. Other terahertz radiation detection applications include plasma diagnostics [7], military radar and radiometry [8], chemical spectroscopy and analysis [9], bio-particle reorganizations [10, 11], security screening and terahertz imaging [12-14].

Two types of detection schemes are used in the terahertz regime, "direct" or incoherent detection (only magnitude information) [15, 16] and "heterodyne" or coherent detection (both magnitude and phase information). At terahertz frequencies, heterodyne detection (also called "mixing") typically exhibits higher sensitivity and greater dynamic range than direct detection schemes. Heterodyne receivers are frequency translators that convert a high-frequency ("RF") signal to a lower-frequency (called the intermediate frequency or " $\mathrm{IF}^{\prime}$ ) band. The output IF signal is a replica of the high-frequency signal and preserves both the magnitude and phase information of the original signal.

Currently, THz heterodyne detectors (or receivers) based on superconducting niobium SIS mixers offer the highest sensitivity. However, these devices exhibit a gap frequency around $700 \mathrm{GHz}$ at which the photon energy exceeds the binding energy of the Cooper pairs in the superconductor, directly converting the superconductor into its normal, lossy state. Above the gap frequency, superconducting hot-electron bolometer (HEB) mixers become much attractive since the operation of HEB does not depend on the tunneling of quasi-particles across the insulator.

Receivers employing Schottky diodes are routinely used in laboratories at room temperature. However, they require relatively large LO power $(\sim 1-10 \mathrm{~mW})$, which is unpractical for space-borne applications and difficult to implement for receiver arrays. HEB receivers normally require very low local oscillator $(\mathrm{LO})$ power $(\sim 100 \mathrm{nW})$, while providing higher 


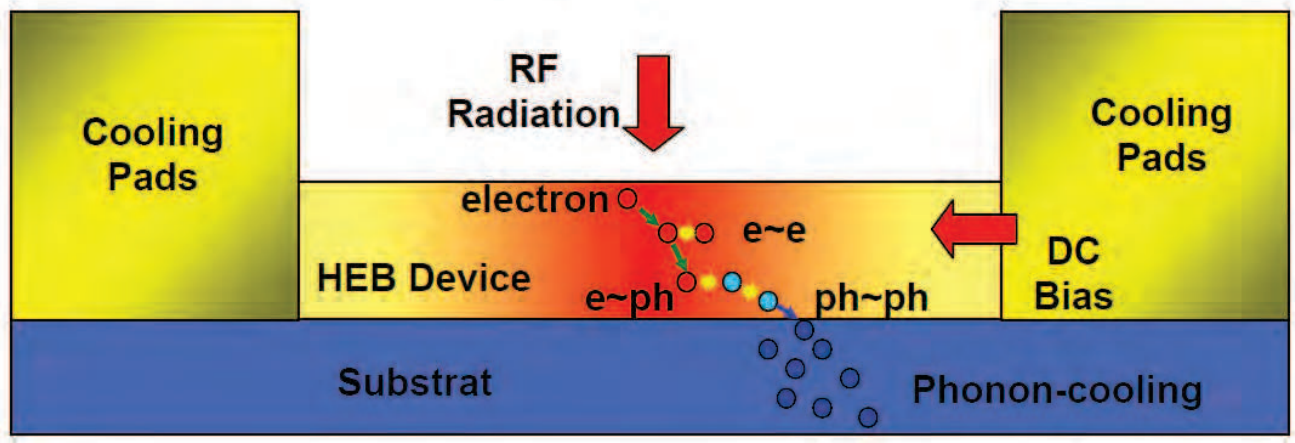

Fig. 1. The operation of HEB devices, the electron-electron cooling/scattering, electron-phonon cooling/scattering and phonon-phonon cooling/scattering are denoted as e-e, e-ph and ph-ph, respectively.

sensitivity than Schottky diode receivers, thus became an attractive research interest in recent years.

In this chapter, the basic theory/mechanism for HEB devices working as THz heterodyne mixing/detection elements will be first introduced, followed by a literature review on what have been done so far for quasi-optical (lens-coupled) HEB THz mixers. The state-of-the-art HEB mixer performance will be summarized. After that, the simulation, design, fabrication and characterization of $585 \mathrm{GHz}$ hot-electron mixers based on annular slot antennas will be presented. On the basis of the single element mixer design, the realization and performance of one-dimensional and two-dimensional HEB mixer focal-plan arrays will be discussed.

\section{Hot-electron bolometers and mixers}

Hot-electron bolometer is a type of bolometric thermal detector that senses changes in temperature $(T)$ through a change in resistance $(R)$. Energy absorbed is distributed to an electron subsystem with a heat capacity $C$, and a thermal conductivity to a heat sink, $G$. Typically, the absorber (also the electron subsystem) is connected to a heat sink with a bath temperature $T_{b}$. The HEB voltage sensitivity $S$ can be found by [17]:

$$
S=I_{\text {bias }} \frac{d R}{d T} \frac{1}{G \sqrt{1+\omega_{I F^{2} \tau^{2}}}}
$$

where $I_{\text {bias }}$ is biasing current, $\omega_{I F}$, the IF frequency, and $\tau$ the thermal response time given by $\tau=C / G$. Note that in equation (x.1) the sensitivity is directly proportional to the change in resistance with temperature. Superconducting HEB's have a very sharp $d R / d T$ slope around the critical temperature. Thus extremely sensitive receivers based on those devices can be achieved. Also, the heat capacity of electrons is much smaller than that of the lattice phonons. At low temperatures, coupling between the electrons and lattice is relatively weak, absorbed RF energy effectively heats only the electrons. Because coupling to the lattice phonons is weak, the lattice does not contribute much to the overall specific heat of the device, thus allowing faster cooling and broader bandwidth operation. This also results in a higher sensitivity, according to equation (x.1). 
As seen in Fig. 1, heated electrons in the subsystem exchange absorbed energy (from RF power) through electron diffusion or phonon scattering. The characteristic electron-phonon scattering length $L_{e-p h}$ (also called thermal heating length $\lambda_{t h}$ ) is the mean free path before an inelastic electron-phonon scattering event takes place. If the bolometer length is larger than $\lambda_{t h}$, the dominant cooling mechanism is phonon scattering, and the bolometer is called a "phonon-cooled" HEB. If the bolometer length is in the order of electron diffusion length $L_{e-e}$, but is less than $\lambda_{t h}$, the bolometer is then called a "diffusion-cooled" HEB, and the dominant cooling mechanism is then diffusion of hot electrons to cooling pads connected to the device. As a result, the thermal response time for a diffusion-cooled HEB ( $\tau_{e-e} \sim 0.1 \mathrm{~ns}$ ) is shorter than that of phonon-cooled HEB $\left(\tau_{p h} \sim 1 \mathrm{~ns}\right)$, resulting in a broader 3-dB intermediate frequency (IF) bandwidth $\left(f_{I F, 3-d B}=(2 \pi t)^{-1}\right)$ [17].

As discussed above, the small size $(\sim 100 \mathrm{~nm})$ and fast response of superconducting HEBs allow them to be operated as heterodyne mixers (IF envelope detectors) rather than as simple power measuring devices. Fig. 2 shows how the HEB device operates as a mixer. The HEB absorbs power from the LO and RF signals. The device then warms, causing a portion of the HEB microbridge to become resistive $\left(L_{H}\right)$. When operated in the optimal regime, the size and electron temperature of the resistive portion is very sensitive to the instantaneous power level. The HEB bridge cools by losing energy to the surrounding environment through various mechanisms as discussed above. The heating and cooling of the HEB follows the envelope of the LO and RF signals, which is at the IF (LO-RF or RF-LO) beat frequency. The resistance of the device, therefore, follows the beat signal between the $\mathrm{LO}$ and RF and thereby generates the IF when the HEB is current or voltage biased. This mixing mechanism allows HEBs to generate IF signals very effectively utilizing the material's superconductive/resistive transition at its critical temperature $\left(T_{c}\right)$.

The canonical architecture for heterodyne mixing is shown in Fig. 3. The mixer usually comprises an electronic device (here will be HEB) with highly-nonlinear current-voltage (I-V) characteristics and its associated parasitic and matching networks. This is usually followed by a low-pass filter (LPF) and low noise amplifier (LNA) which, together, make up the IF chain. The frequency translation properties of a nonlinear element are readily illustrated by examining a square-law device with current-voltage relation: $I=A V^{2}$, with A being a constant dependent on the device. The RF input and LO can be represented as a superposition of sinusoidal functions at two slightly different frequencies, $f_{L O}$ and $f_{R F}$ as expressed in equation X.2.

$$
V_{L O}=V_{1} \sin \left(2 \pi f_{L O} t\right), V_{R F}=V_{2} \sin \left(2 \pi f_{R F} t\right)
$$

When applied to the device, these signals will generate outputs at DC, $f_{R F}+f_{L O}$ and $f_{R F} f_{L O}$. Applying the square-law relation to the applied voltage yields the total current signal flowing through the mixer as

$$
I=\alpha\left[V_{D C}+V_{1} \sin \left(2 \pi f_{L O} t\right)+V_{2} \sin \left(2 \pi f_{R F} t\right)\right]^{2}
$$

This output signal, after filtering and amplification, will be proportional to $V_{1}$ and $V_{2}$, but is translated to a frequency of $f_{I F}=f_{R F}-f_{L O}$, as shown in equation X.4: 


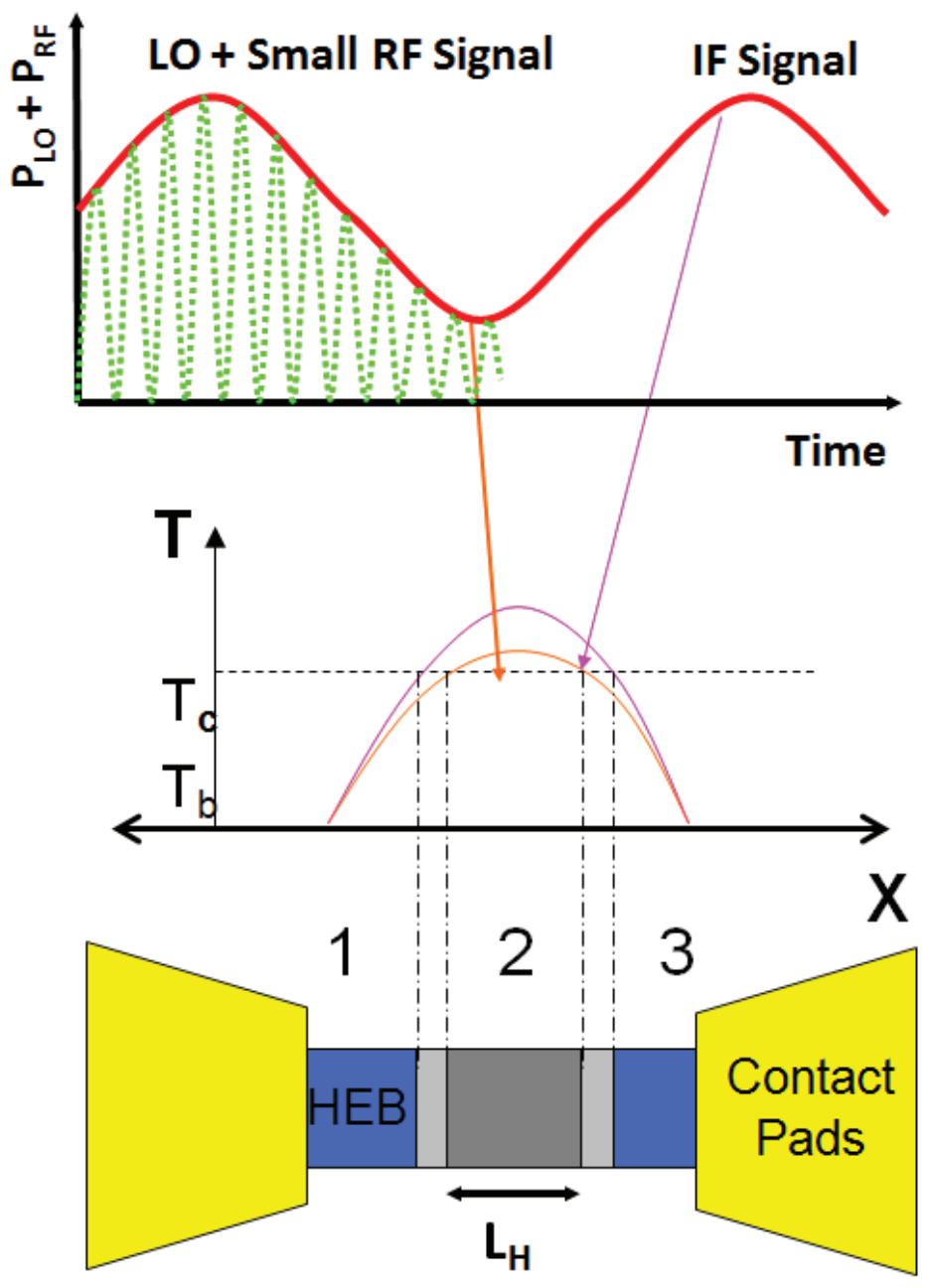

Fig. 2. The operation of a superconducting hot-electron bolometer device as a heterodyne mixer (figure reproduced from [1].

$$
V_{\text {OUT }}=V_{1} V_{2} \cos \left(2 \pi f_{I F} t\right)
$$

Consequently, information contained in the signal at the RF frequency is down-converted to the IF band, which is more readily processed. This particular receiver scheme responds to both the RF signal and its image, $f_{I M}$, at $f_{I F} \pm f_{L O}$. Such a receiver is called a double sideband (DSB) mixer. In some cases, a band-pass filter (BPF) may be placed in front of the mixer, and reject the unwanted sideband. Thus the mixer is operated in single side band (SSB) mode and either the lower side band (LSB) or upper side band (USB) is chosen, depending on the overall application. Usually DSB mixers are undesirable because noise enters the image port 


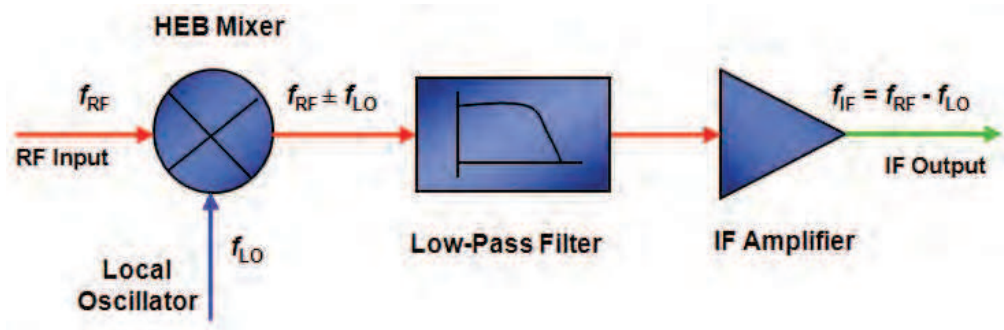

(a)

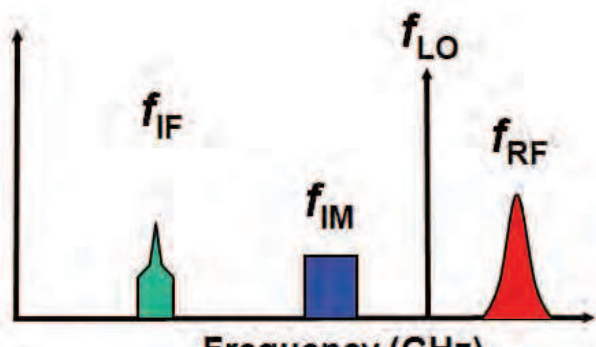

Frequency $(\mathrm{GHz})$

(b)

Fig. 3. Heterodyne mixing schematics: (a) Receiver diagram, (b) Signal spectrum.

and contributes to mixer noise. However, they are the most common type since it is difficult to implement image rejection filters at $\mathrm{THz}$ frequencies.

\section{Lens-antenna coupled HEB THz mixer}

\subsection{Lens-coupled antenna configuration for $\mathrm{THz}$ detection and imaging}

As the frequency increases into the submillimeter-wave and terahertz region, the detection of RF signals becomes challenging largely because of difficulties associated with extending classical microwave technologies and techniques to this frequency regime [18]. Loss introduced by metallic waveguides that are generally used at microwave frequencies increases with frequency (e.g. the surface resistivity of the waveguide is proportional to $\sqrt{\omega \mu / 2 \sigma}$ ). Also, the cost and tolerances associated with machining small structures are difficult to fulfill in fabricating waveguide structures with traditional milling techniques for use at terahertz frequencies [18]. Although microfabrication techniques using DRIE or SU-8 have been applied to the submillimeter-wave and terahertz waveguide structures [19], an alternative approach $U^{\circ}$ quasi-optics is attractive, particularly as the wavelength of the signals approach the infrared region of the spectrum. Quasi-optical technology combines both the microwave and optical 


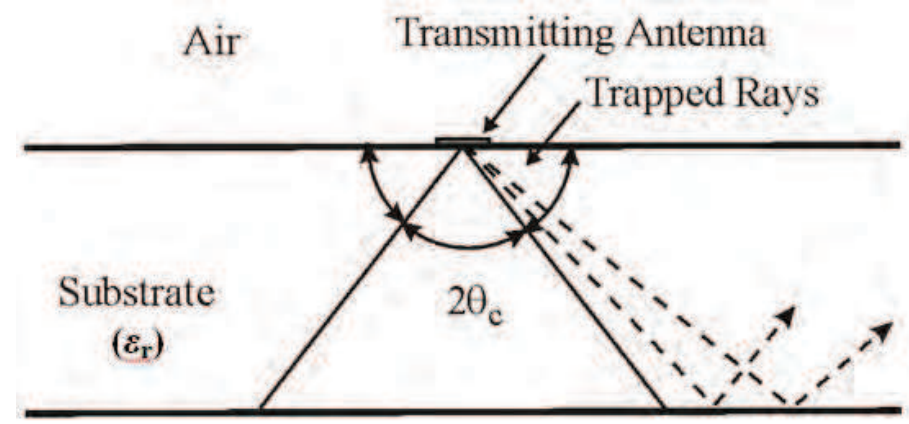

Air

(a)

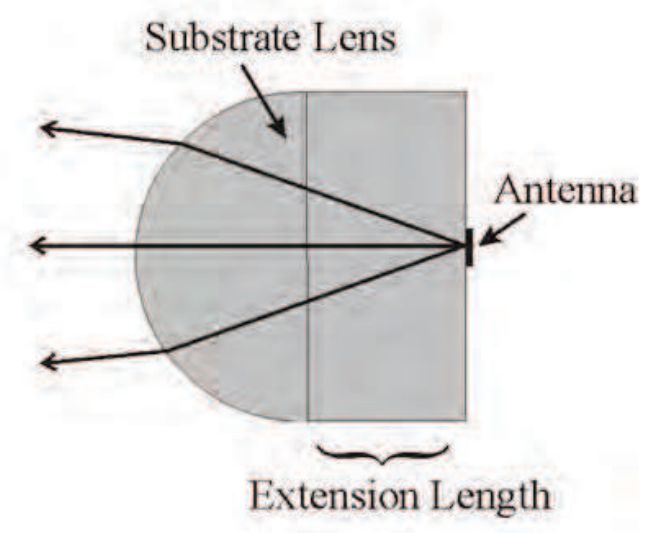

(b)

Fig. 4. (a) Transmission antenna on a dielectric substrate showing the generation of surface waves, reproduced from [20]. (b) Lens-antenna coupled $\mathrm{THz}$ detector and imaging configuration.

techniques, making it quite suitable and useful for applications in submillimeter-wave and terahertz region.

For submillimeter-wave and terahertz applications such as imaging arrays, that make use of quasi-optical technology, detectors or detector arrays are typically fabricated on a dielectric substrate such as GaAs, silicon, or quartz. The signal to be detected is first focused onto the substrate by an objective lens, and then illuminates the detectors from the dielectric side. In this way, the coupling structures (or antennas), should exhibit the maximum receiving directivity. However, antennas on dielectric substrates generate trapped surface waves, which unavoidably decrease the antenna efficiency and increase the cross-talk between adjacent antennas in an array [20]. This can be understood on the basis of simple geometric optics with 
a transmitting antenna shown in Fig. 4(a) [20]. The transmitting antenna lies at the upper air-dielectric interface, and radiates into the substrate. From the ray-tracing point of view, only the rays with a radiation angle of $\theta<\theta_{c}$ can go through the lower air-dielectric interface, where $\theta_{c}=\arcsin \left(\epsilon_{r}\right)$ is the well-known critical angle. Other rays are completely reflected and trapped as the surface waves. The power that is converted to surface waves can be very large, dramatically reducing the antenna transmission/receiving efficiency. In addition, due to the existence of trapped surface waves, crosstalk between adjacent antennas in an imaging array can be significant increased, limiting the resolution that can be achieved by an imaging array.

The simplest method for solving the problem of surface wave excitation is to mount a dielectric lens to the detector/antenna substrate as shown in Fig. 4(b). If the lens and the substrate have the same dielectric constant, most of the incident rays are then nearly normal to the air-dielectric interface, eliminating total internal reflection at this interface. An imaging architecture, the so called "reverse-microscope" concept, is based on the above consideration and was first proposed by Rutledge and Muha in 1982 [13]. In this architecture, both an objective lens and a substrate lens are utilized for coupling incident radiation $(\mathrm{THz})$ onto the antenna structure, resulting in an approach capable of diffraction-limited imaging. In our research, an extended hemispherical substrate silicon lens is utilized (see Fig. 4(b)), and the antenna coupled HEB mixers and mixer imaging arrays are fabricated based on this "reverse-microscope" configuration. This architecture will be further discussed later in this Chapter.

\subsection{Lens-coupled THz antenna and mixer performance}

As discussed above, at terahertz frequencies, RF circuits based on waveguide transmission media are more difficult to implement and quasi-optical techniques become an attractive alternative. To couple the incident RF power to the nonlinear HEB mixing element, planar antennas such as bow-tie [21], double-slots [22, 23], spiral [24], and log-periodic [25], mounted on lens-coupled dielectric substrates are frequently utilized as shown in Fig. 5. However, Bow-tie antennas have a number of drawbacks in the $\mathrm{THz}$ region since they are not compact for single imaging element design and exhibit antenna patterns with maximum off the antenna bore-sight. $\mathrm{THz}$ mixer designs using other antenna geometries also have not yet been suitable for high resolution imaging applications. Annular-slot antennas are one type of planar structure that can efficiently couple incident power to a device located at the feed point. They have very compact and symmetric geometries, making themselves good candidates for large scale imaging arrays. Moreover, this antenna structure can be easily scaled up to submillimeter-wave and terahertz region. In the following sections, we will focus on $\mathrm{THz}$ HEB mixers and focal-plane arrays based on annular-slot antennas.

State of the art HEB mixer performance compared to SIS and Schottky mixers are summarized in Fig. 6 [26]. Typically, receiver noise temperature in the order of 10 times of quantum limit has been achieved with HEB mixers. A theoretic analysis for the noise temperature of a superconducting HEB mixer is presented in [27]. Johnson noise and thermal fluctuation noise are the two main noise sources. For an optimum operation of a niobium device, the noise contribution from the above two mechanisms can be as low as $0.4 \mathrm{~K}$ from Johnson noise and $22 \mathrm{~K}$ from thermal fluctuation noise. 

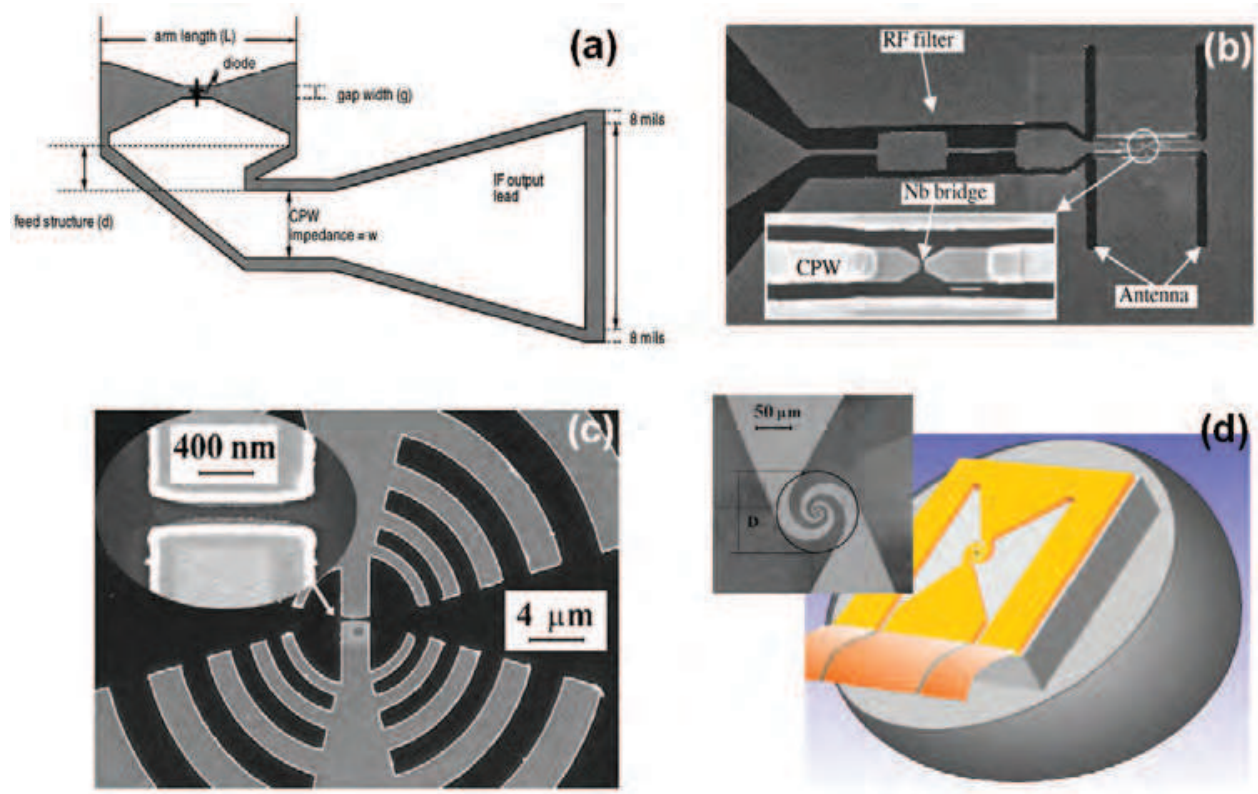

Fig. 5. Lens-coupled (a) bow-tie antenna [21], (b) double-slot antenna [22, 23], (c) log-periodic [25] and (d) spiral antenna [24] for THz HEB mixer design.

\subsection{Superconducting hot-electron bolometer device fabrication}

The HEB fabrication used in this research is based on the UVa EBL process developed by Bass et al [2]. The fabrication process includes several main steps: (1) base layer definition; (2), e-beam lithography (EBL) process to define the HEB length and width; (3) Reactive-Ion Etch (RIE) to remove unwanted metal, and (4), the passivation process.

The base layer definition begins with the deposition of a $\mathrm{Nb} / \mathrm{Au}(10 \mathrm{~nm} / 10 \mathrm{~nm})$ bi-layer onto a square silicon wafer (high resistivity, $1.8 \mathrm{~cm} \times 1.8 \mathrm{~cm}$ ). This niobium base layer is used for defining the superconducting HEB micro-bridge, which is the most critical step in the mixer fabrication. To optimize the superconducting properties of this layer, a multi-target sputtering system is utilized and the deposition conditions (e.g. base pressure $\sim 10^{-8}$ Torr) are carefully controlled to achieve a low-stress niobium thin film. A film thickness of $10 \mathrm{~nm}$ is chosen to yield a sheet resistance of $35 \Omega$ /square at the normal state and a critical temperature of $\sim 5.6$ $\mathrm{K}$ [2]. A $10 \mathrm{~nm}$ gold over-layer is deposited with the same sputtering system to protect the superconducting niobium from oxidization during the subsequent fabrication processes.

After deposition of the $\mathrm{Nb} / \mathrm{Au}$ bi-layer over the entire wafer, photoresist (AZ5206) is spun on top of the wafer as a sacrificial layer. The burn-off mask is first applied to eliminate the edge head, followed by the base layer lithography. Ti and Au (5 nm/200 nm) layers are then deposited onto this resist stencil using the e-beam evaporator system. Finally, a lift-off process is performed using NMP and propylene glycol (P-Glycol) heated to $110^{\circ} \mathrm{C}$ to lift off (remove) the unneeded metals on the sacrificial layer, thus leaving the Ti/Au RF circuits and EBL alignment/focusing markers on the wafer. It is important to note that a gap in the $\mathrm{Ti} / \mathrm{Au}$ 


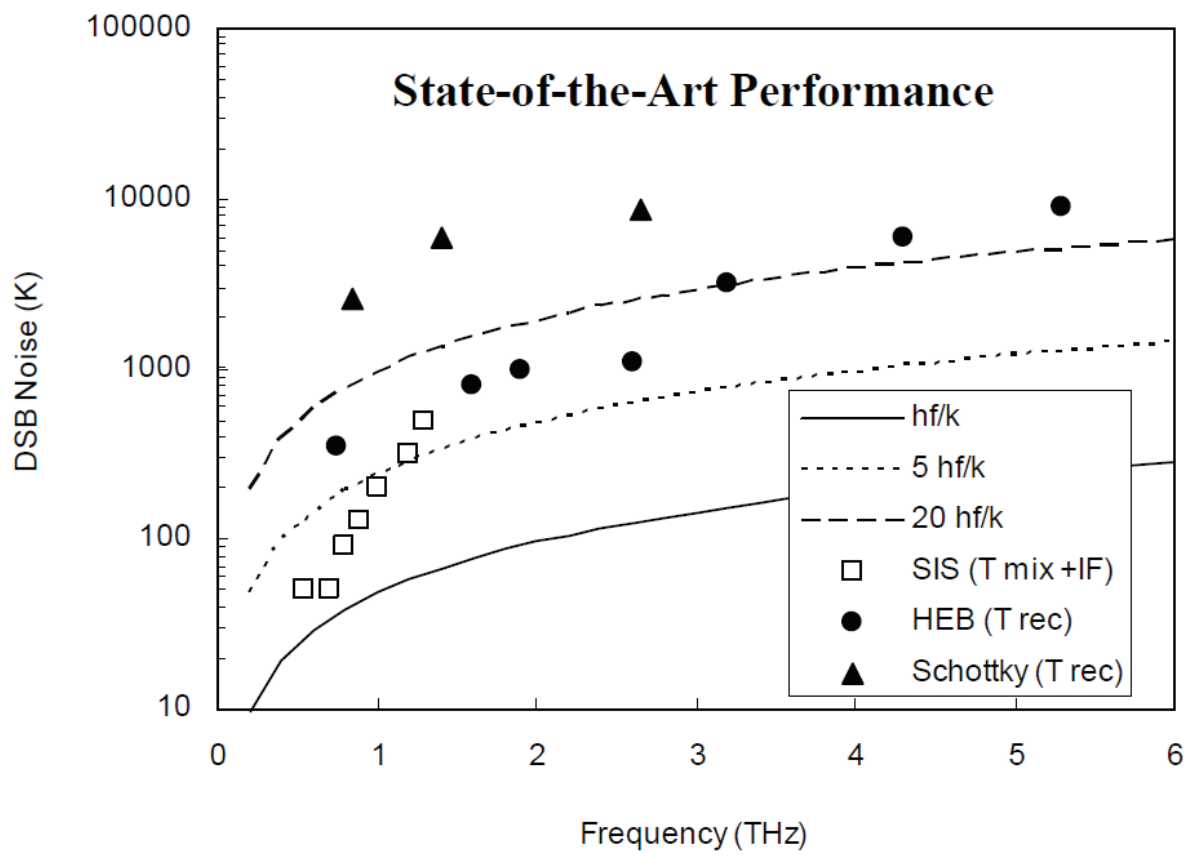

Fig. 6. State-of-the-art performance for SIS, HEB and Schottky mixers. This figure is reproduced from [26]

bilayer is left for the subsequent deposition of the HEB cooling pads and definition of the underlying $10 \mathrm{~nm}$ thick $\mathrm{Nb}$ HEB bridge.

After the base layer is defined, the mixing element - the HEB bridge - is fabricated using a two-step EBL process (see process flow in Fig. 7). In the first EBL step, a $200 \mathrm{~nm}$ thick bilayer PMMA (950/495) is spun on the base layer as the resist structure. The high molecular weight (950) PMMA on top is used for maximizing EBL resolution, while the low molecular weight (495) PMMA generates an undercut beneath the patterns after development, thus eliminating metal sidewalls during the following deposition process. This facilitates the lift-off process.

The HEB device cooling pads are then directly written by an electron-beam controlled by the Nano-Pattern Generation System (NPGS). During this "direct-writing" process, the EBL alignment markers on the base layer are applied to precisely "write" the patterns. Once again, prior to each HEB pattern writing, the focusing markers are utilized to adjust the SEM parameters to improve the device fabrication uniformity. To produce a HEB device length of around $200 \mathrm{~nm}$, the cooling pads are designed using DesignCAD with a separation of $290 \mathrm{~nm}$.

After developing the PMMA using a 1:3 mixture of MIBK and IPA, a trilayer of $\mathrm{Ti} / \mathrm{Au} / \mathrm{Ti}$ $(10 \mathrm{~nm} / 50 \mathrm{~nm} / 10 \mathrm{~nm})$ is evaporated. The first Ti layer serves as the adhesion layer and the second protects the Au during a subsequent RIE process. After a lift-off process using TCE heated to $70{ }^{\circ} \mathrm{C}$, the HEB cooling pads are realized and the $\mathrm{Nb}$ microbridge length is defined. SEM pictures in Fig. 8(a) clearly show the designed and fabricated HEB cooling pads patterns. Each cooling pad overlaps both the $\mathrm{Nb} / \mathrm{Au} \mathrm{HEB}$ device layer film and the $\mathrm{Ti} / \mathrm{Au} \mathrm{RF}$ line and 


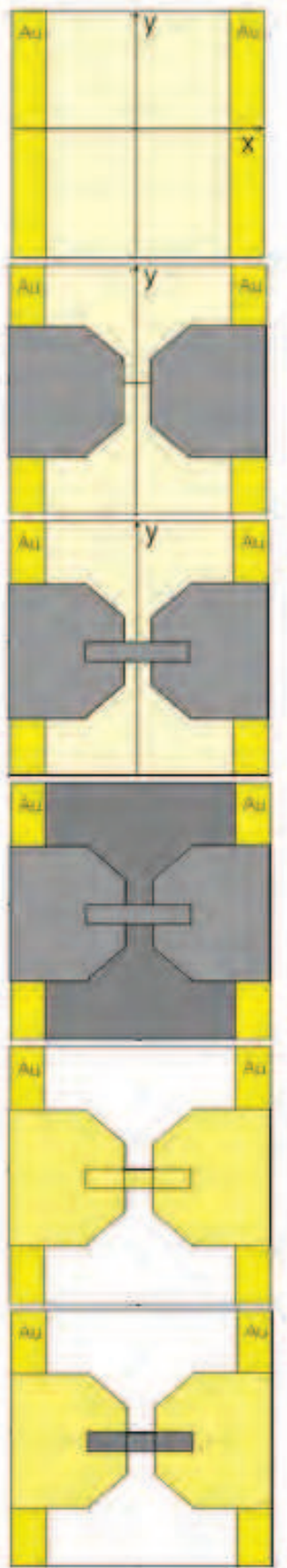

$\mathrm{x}$-section
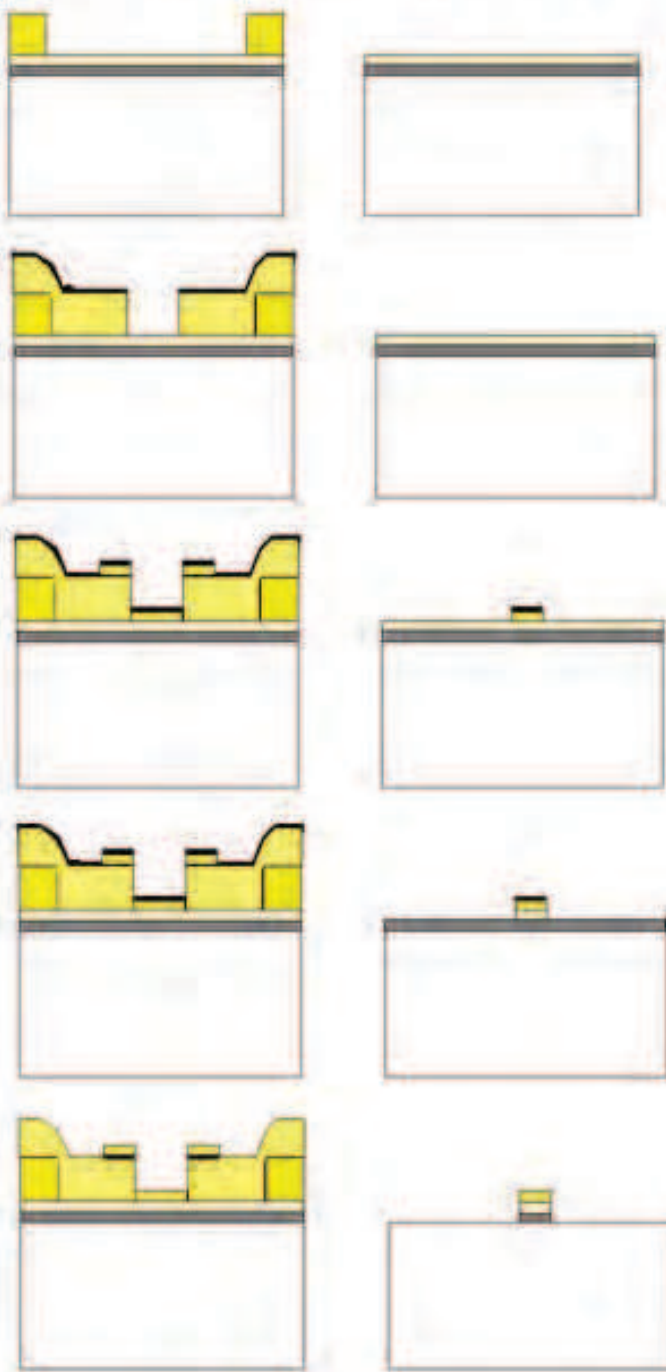

(c)

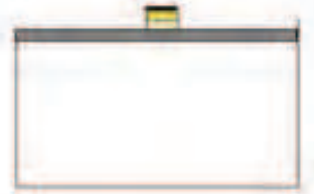

(d)

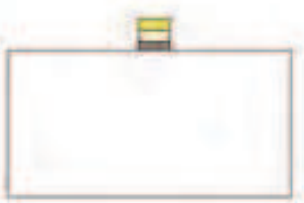

(e)
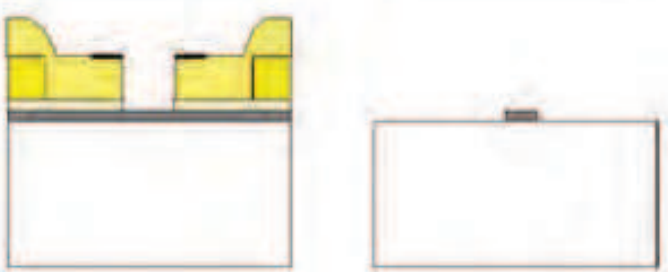

(f)

Fig. 7. A typical hot-electron bolometer device fabrication process using e-beam lithography. 

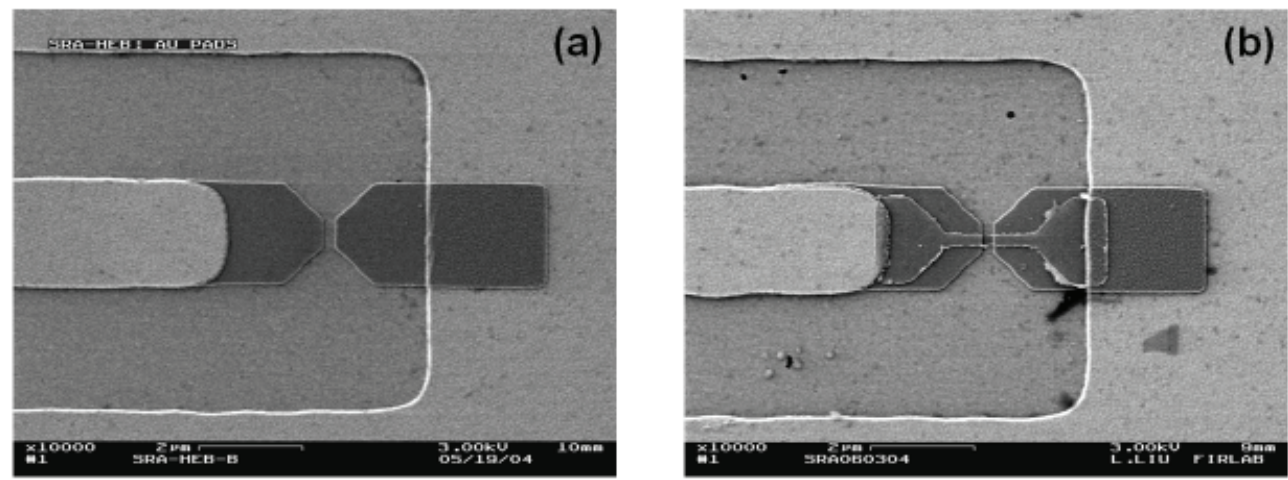

Fig. 8. Hot-electron bolometer device fabrication: (a)SEM picture showing the device cooling pads defining the HEB length, and (b)SEM picture showing the HEB bridge masks, defining the device width.

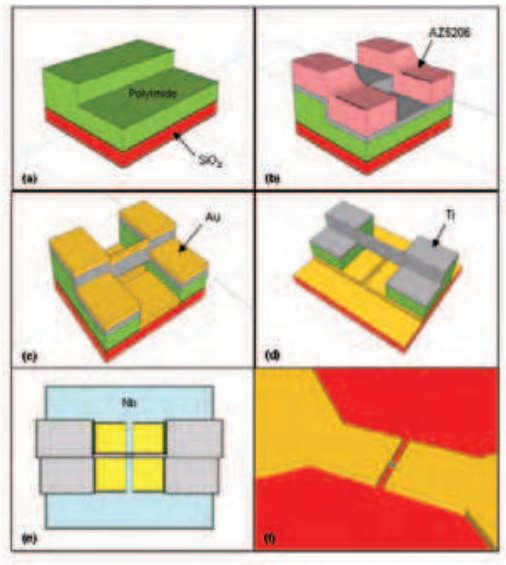

(a)

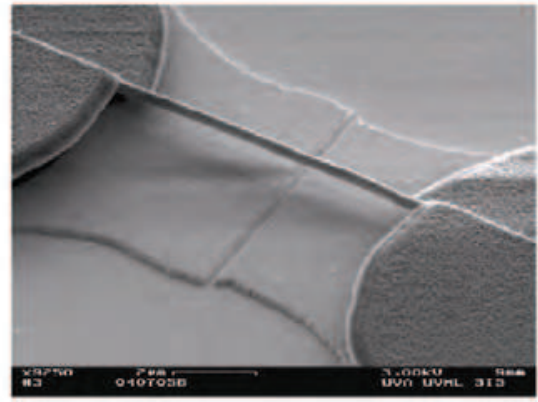

(b)

Fig. 9. Hot-electron bolometer device fabrication using the Ti-line technique without EBL process: (a) fabrication process, and (b) SEM picture of a suspended Ti-line (reproduced from [28]).

essentially serves to continue the $\mathrm{Ti} / \mathrm{Au}$ contacts on each side in order to precisely define the length of the bridge.

During the second EBL step, the HEB bridge etch mask is first patterned by the NPGS with a single layer PMMA (950). The bridge width is designed to be $200 \mathrm{~nm}$ and $140 \mathrm{~nm}$, for secheme-I and scheme-II structures, respectively. A Au/Nb $(20 \mathrm{~nm} / 20 \mathrm{~nm})$ bilayer is deposited, and after lift-off, the bridge etch mask is left which also overlap part of the cooling pads as seen in Fig. 8(b). This $\mathrm{Au} / \mathrm{Nb}$ bridge serves as a mask in the RIE processes, described below that defines and reveals the HEB $\mathrm{Nb}$ bridge width.

Reactive-ion etches (RIE) are therefore performed to define the width of the bridge to remove the unnecessary metal layers. First, an Ar-RIE is performed and after this etching, the 
open-field over-layer gold $(10 \mathrm{~nm})$ in the RF circuits is removed, exposing the underneath niobium layer (Fig. 7(d)). An $\mathrm{SF}_{6}$-based RIE is then used to etch the exposed niobium layer both in the open field and on the top of the HEB bridge (Fig. 7(e)), followed by another Ar-RIE to remove the final layer of $\mathrm{Au}$ on top of the bridge, which leaves only a niobium bridge between the gold cooling pads (Fig. 7(f)). The conditions of the above etch steps are precisely controlled so that the etching rates are appropriate for operation. This is very important since over-etching typically significantly changes the device resistance and insufficient etching can results in total failure.

After the RIE process is finished, the HEB bridge is defined and the superconducting niobium is exposed to the ambient atmosphere. To prevent it from contamination and oxidization, a 300 $\mathrm{nm}$ germanium layer is e-beam deposited across the entire wafer. General photolithography is applied to have square photoresist patterns $(20 \mu \mathrm{m} \times 20 \mu \mathrm{m})$ only in the HEB area. A subsequent $\mathrm{SF}_{6}$-based RIE is performed to remove the exposed germanium, leaving square germanium covering the HEB devices. Since germanium is a semiconductor, carrier freeze-out and the low energy associated at sub-millimeter wave and terahertz frequencies prevents this material from shorting the RF circuits, while it provides a protective passivation layer for the superconducting niobium HEB devices.

Although the EBL-based HEB fabrication process is used in this research for prototype demonstration, it is strongly depends on the operator's skills and quite time-consuming. Thus the device uniformity is not perfect, and the process is not suitable for the fabrication of focal-plane arrays with large number of imaging elements. The so called "Suspended Sidewall Nano-Patterned Stencil" (SSNaPS or Ti-line) process as shown in Fig. 9 [28] is then strongly preferred. Using this method, the whole fabrication can be done without relying on those expensive e-beam or ion-beam facilities, allowing more research organizations to make and test HEB devices. In addition, quick fabrication of large quantities of HEB's on one wafer with perfect uniformity becomes readily realizable, making this SSNaPS process a good choice for making large focal plane arrays.

\subsection{Single THz HEB mixer element based on annular-slot antennas}

Annular-slot antennas are one type of planar structure that can efficiently couple incident power to a device located at the feed point. Thus these antennas have been studied in detail for a variety of microwave and millimeter-wave circuits including mixers [29] and frequency doublers [30]. Moreover, the annular slot antenna (ASA) provides a relatively compact geometry, which is attractive for high resolution imaging array applications [31], [32]. Fig. 10 illustrates the basic geometry of an annular slot with single feed-point and lying on a dielectric half-space with dielectric constant of $\epsilon_{r}$. At its resonant frequency, the circumference of the annular slot is equal to nearly one wavelength, resulting in a sinusoidal electric-field distribution around the slot. As seen in Fig. 10(a), with incident field polarized along the $y$-direction, the electric-field in the slot has a maximum amplitude at $\varphi=90^{\circ}$ and $\varphi$ $=270^{\circ}$, with nulls (or virtual shot-circuits) at $\varphi=0^{\circ}$ and $\varphi=180^{\circ}$. Thus the far-field radiation pattern has its E-plane along the $y$-axis, and $\mathrm{H}$-plane along the $\mathrm{x}$-axis. In summary, the annular slot features properties leading to its choice as a mixer coupling structure suitable for imaging array applications.

In this research, high-resistivity silicon is chosen as the mixer fabrication substrate because it has low loss $(\sim 2 \mathrm{~dB} / \mathrm{cm})$ and high dielectric constant $\left(\epsilon_{r}=11.7\right)$, which results in a high 


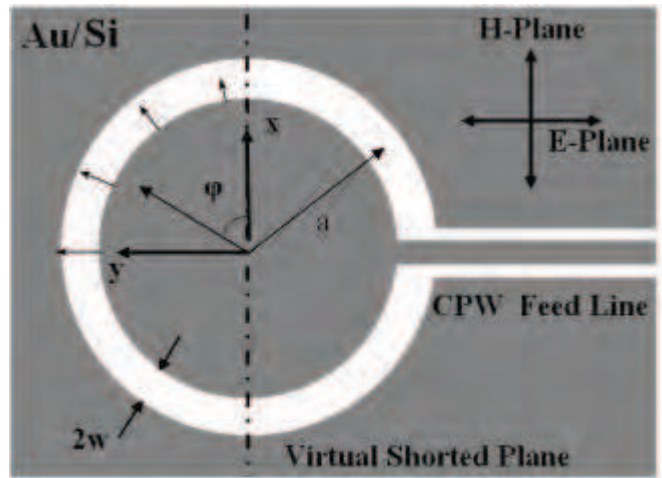

(a)

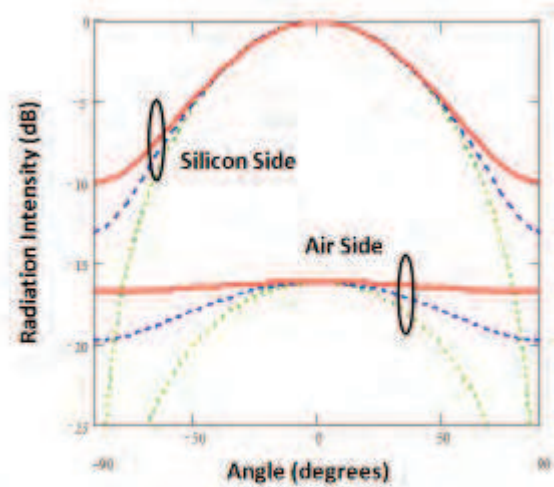

(b)

Fig. 10. (a) Annular-slot antenna on silicon substrate with circumference of one guided wavelength, for operation at $585 \mathrm{GHz}$. (b) Calculated radiation intensity of the $585 \mathrm{GHz}$ annular-slot antenna.

directivity and efficiency for the receiving antenna [22]. The annular slot antenna is designed to operate at $585 \mathrm{GHz}$ and has a radius, a, of $36 \mu \mathrm{m}$ and a slot width, $\mathrm{w}$, of $2.6 \mu \mathrm{m}$, as shown in Fig. 10(a). The annular slot antenna radiation pattern at $585 \mathrm{GHz}$ in the silicon substrate and in the air side, for $\varphi=0, \pi / 4$, and $\pi / 2$ planes are plotted in Fig. 10(b). As can be seen, the radiation intensity on the silicon side is much larger than that on the air side, resulting in a higher antenna directivity. The ratio of power radiated into the half-spaces is $\sqrt{\epsilon_{r-S i}}{ }^{3}$ : ${\sqrt{\epsilon_{r-a i r}}}^{3}(\sim 40: 1)$, according to [20].

An ADS Momentum simulation of the annular-slot antenna to determine its impedance has been performed, and the results show that the designed annular slot antenna has a bandwidth of approximately $100 \mathrm{GHz}$ (giving a fractional bandwidth of $16 \%$ ). At $585 \mathrm{GHz}$, the real and imaginary parts of the antenna input impedance are $100 \Omega$ and approximately $0 \Omega$, respectively. Consequently, impedance matching to the devices is relatively straightforward because the superconducting HEB bridge is essentially a purely resistive device. A d-HEB with resistance as high as $100 \Omega$, however, requires nearly 3 squares of $\mathrm{Nb}$ thin film $(10 \mathrm{~nm}$ thick with a sheet resistance of $35 \Omega$ /square in the normal state). Hence, the resulting device length approaches that of the inelastic electron-phonon mean free path, $L_{e-p h}$. Because the resolution of the Nanometer Pattern Generation System (NPGS) employed at the University of Virginia is approximately $100 \mathrm{~nm}$, the resulting device could well exceed the maximum length for diffusion cooling. As a result, a quarter-wavelength impedance transformer is employed to match the $100 \Omega$ antenna impedance in the mixer design.

Another consideration in the receiver design using the lens-coupled antenna configuration is the Gaussian coupling efficiency and antenna system directivity. Analysis for double-slot antennas on extended hemispherical and elliptical silicon dielectric lens was carried out using a ray-tracing technique by Filipovic and Rebeiz et al. in 1993 [22]. Their results demonstrate that the directivity strongly depends on the extension length of the lens, L (see Fig. 4), and reaches a maximum at a particular value of $\mathrm{L}$, while the Gaussian coupling efficiency maintains a relatively high value and then drops quickly as L increases. Thus, the design goal 
for an imaging array application is a high system directivity for maximum packing density in the focal plane, while maintaining an acceptable Gaussian coupling efficiency. To realize this design goal, the ray-tracing technique used by Filipovic is applied to the case of annular slot antennas on an extended hemispherical silicon lens. The hemispherical silicon lens for this work has a radius $\mathrm{R}=4.5 \mathrm{~mm}$. Radiation patterns of the lens-supported annular slot antenna are then calculated with a numerical computer code based on the ray-tracing technique.

The calculated E-plane patterns for various extension lengths are shown in Fig. 11. It is clearly seen that the main beam of the far-field pattern becomes narrower and then broader with increasing extension length. An extension length of $1600 \mu \mathrm{m}$ is chosen for the highest antenna directivity for imaging array applications. In principle, once the antenna radiation patterns are obtained, the Gaussian coupling efficiency may be calculated with a double integration, as it is a parameter that measures the coupling efficiency between the far-filed patterns of the antenna and the incident Gaussian beam. However, this is very time-consuming due to the complex numerical integrations needed for the computer code. Since the results in [22] are given in terms of the parameters $R / \lambda$ and $L / \lambda$, and these curves are generally applicable to other planer antenna designs with similar radiation patterns. With $\mathrm{L}=1.6 \mathrm{~mm}$, and $\mathrm{L} / \lambda=$ 0.36 , the Gaussian coupling efficiency is estimated to be around $85 \%$.

On the basis of the HEB mixer design and fabrication process described above, single element mixers have been fabricated and shown in Fig. 12(a). The one-square HEB microbridge is integrated at the end of the quarter-wave tranformer with a device length of $240 \mathrm{~nm}$ and a device width of $237 \mathrm{~nm}$, resulting in a device resistance of nearly $35 \Omega$. In this circuit, high/low stepped-impedance low-pass filter is utilized to efficiently block the RF high-frequency signal. To characterize the superconducting properties of the fabricated HEB devices, R-T and I-V curves were taken, and shown in Fig. 12 in which (b) and (c) are the typical results [33]. As seen in Fig. 12(b), a sharp transition between the superconducting state and the normal state is observed at a critical temperature of $T_{\mathcal{C}}=5.4 \mathrm{~K}$ with a transition width $\Delta T_{\mathcal{C}} \sim 0.5 \mathrm{~K}-1.0 \mathrm{~K}$. The typical critical current is measured to be approximately $120 \mu \mathrm{A}$, corresponding to a critical current density of $J_{c} \sim 5.1 \times 10^{6} \mathrm{~A} / \mathrm{cm}^{2}$. This value is comparable to the results reported by other groups [34]. The normal state resistance just above the superconducting transition is nearly $40 \Omega$, implying a thiner $\mathrm{Nb}$ film than intended, resulting in a slightly higher sheet resistance. Fig. 12(c) shows the I-V curves for a HEB device with slightly lower critical current. When the bath temperature increases from $4.2 \mathrm{~K}$ to $4.86 \mathrm{~K}$, the critical current decreases from $70 \mu \mathrm{A}$ to $55 \mu \mathrm{A}$, as anticipated.

To characterize the RF performance of the $585 \mathrm{GHz}$ HEB mixers, a quasi-optical mixer mount was fabricated using oxygen-free copper for operation in a cryogenic vacuum system, as shown in Fig. 13. A tapered circular aperture in the front piece allows incoming radiation to fully illuminate the surface of the silicon lens. The thickness of this piece is designed so that the silicon lens is tightly held to the back piece, ensuring that there is no air gap between the lens and the substrate. A co-planar waveguide (CPW) transmission line was fabricated on a high-resistivity silicon wafter with a thickness of $1.1 \mathrm{~mm}$, to accommodate the output IF signal (Fig. 13) [35]. The IF circuit substrate also serves as the extension length for the lens-coupled annular-slot antenna, and yields a total extension length of nearly $1.6 \mathrm{~mm}$ after mounting to the mixer chip. This extension length is close to the optimum value for maximum antenna directivity according to the ray-tracing results. The HEB mixer chip was mounted to the IF circuit using cryogenic epoxy, and the DC and RF connections were accomplished using gold bonding wires. 

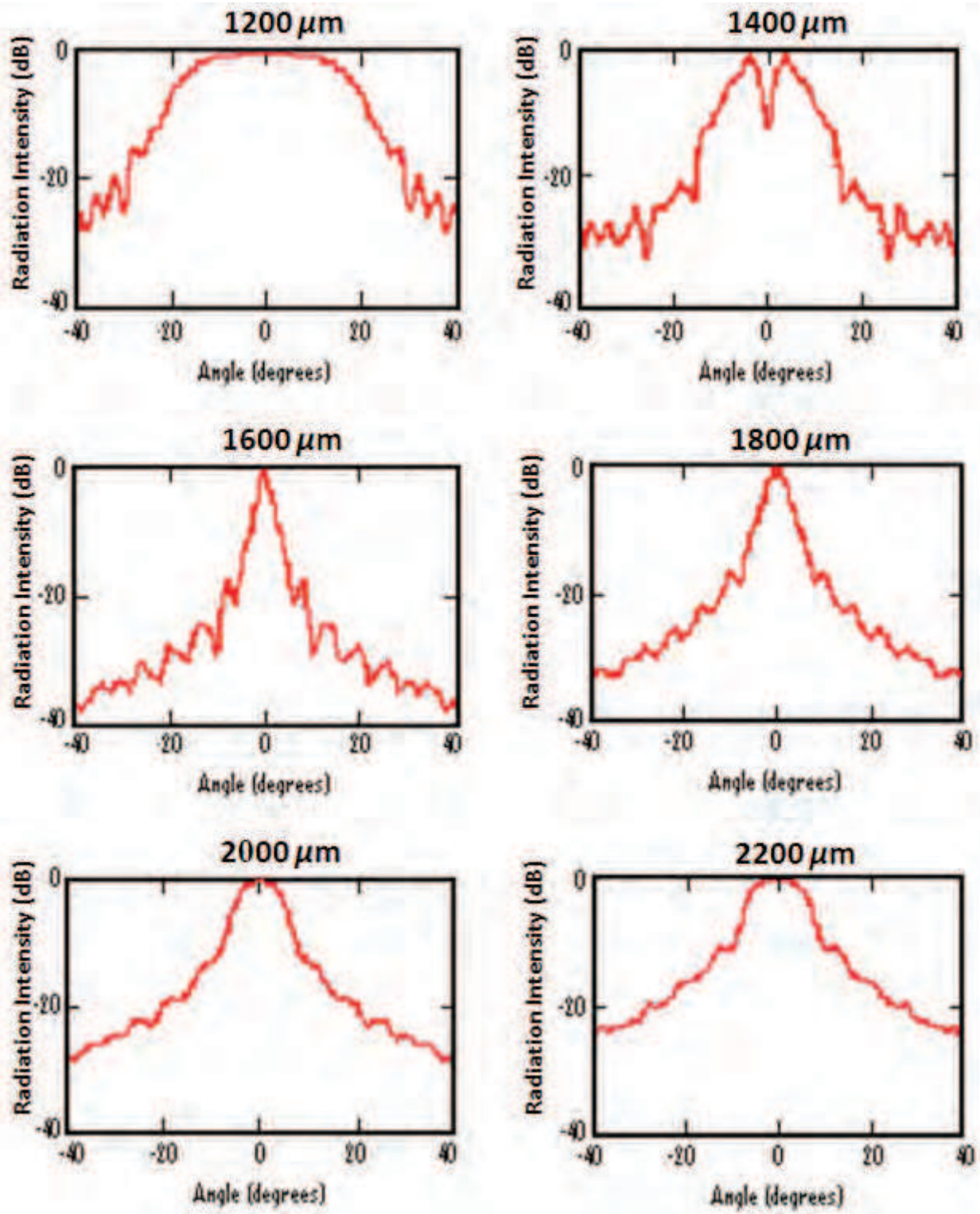

Fig. 11. (a) Annular-slot antenna on silicon substrate with circumference of one guided wavelength, for operation at $585 \mathrm{GHz}$. (b) Calculated radiation intensity of the $585 \mathrm{GHz}$ annular-slot antenna. 

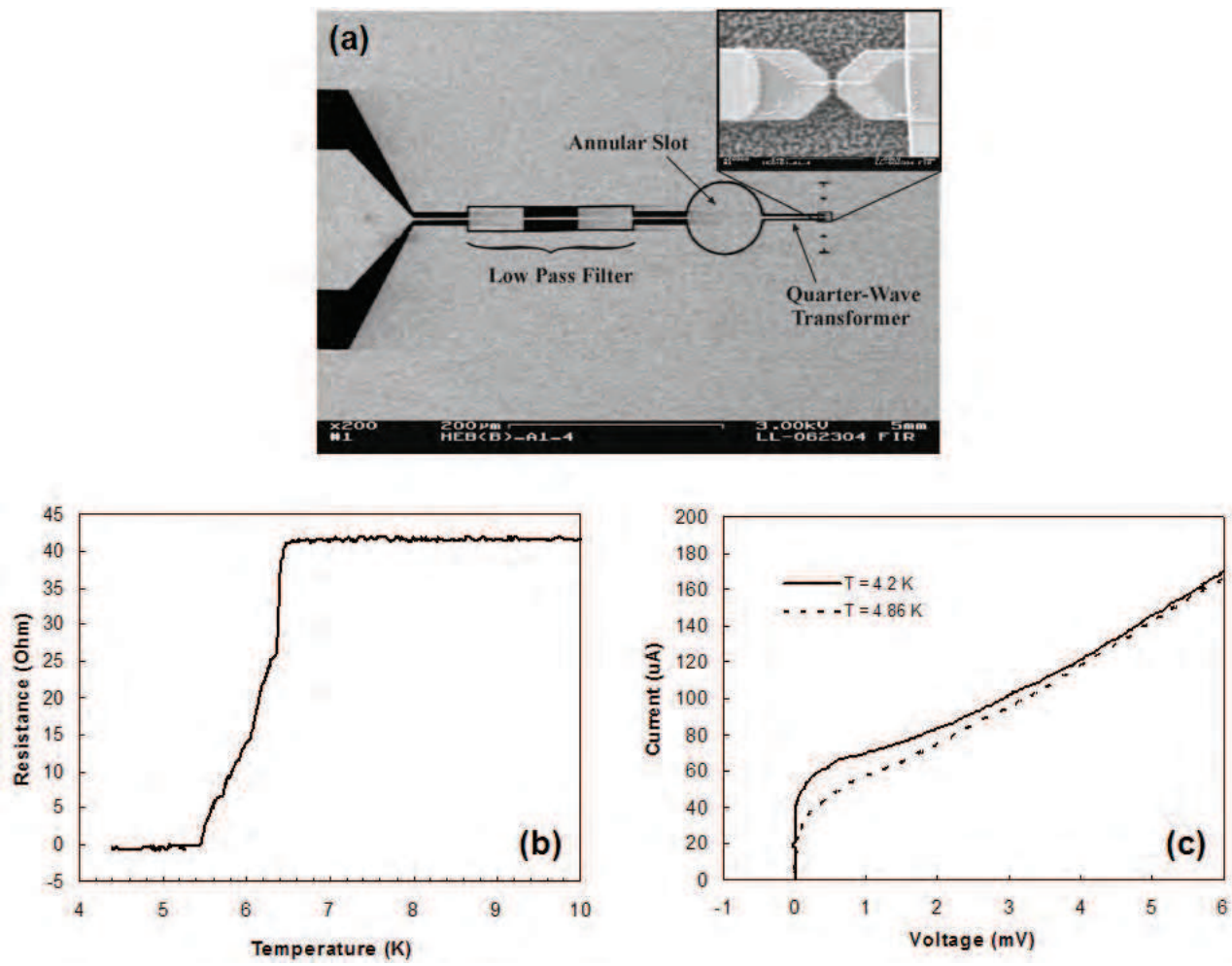

Fig. 12. (a) An annular-slot antenna coupled HEB mixer design utilizing a quarter-wave transformer for impedance matching. (b) R-T curve for a typical HEB device. (c) I-V curves of a HEB device measured at different temperatures.

Once the silver epoxy is cured, the HEB mixer is ready for testing. In this work, an HD-3(8) dewar system is utilized for the single element HEB mixer cryogenic tests and RF measurements. This dewar can be cooled to $4.2 \mathrm{~K}$ with a hold time of around 30 hours. Inside the dewar, the quasi-optical mount with the HEB mixer assembled is installed in front of a Teflon window and is biased through a bias-T. The IF signal is sent to an isolator (Durado 4ICB12-2) and low noise amplifier (LNA) before being fed to the external IF chain. Three temperature sensors are placed at the $4.2 \mathrm{~K}$ plate, LNA, and the quasi-optical mount and the temperatures are displayed outside of the dewar.

The RF measurement setup for characterizing the HEB mixers is shown in Fig. 14. A hot/cold load ( $300 \mathrm{~K} / 77 \mathrm{~K})$ consisting of microwave absorber (Eccosorb) provides the blackbody RF radiation for a system Y-factor measurement. a VDI (Virginia Diodes, Inc.) 576 - $640 \mathrm{GHz}$ FEM (Frequency Extension Module) is employed to provide an available LO power of $0.6 \mathrm{~mW}$ (or $-2.22 \mathrm{dBm}$ ) near $585 \mathrm{GHz}$. The VDI FEM comprises three frequency doublers (D55v2, D100v3, D200) and a WR-1.5 frequency tripler. An Agilent E8247C (0-20 GHz) sweep oscillator and a power amplifier A246-2XW-31 (with a built-in doubler) are used for the signal input. To protect the VDI FEM, a WR-22 variable attenuator is inserted between the power amplifier output and the VDI FEM input. Once the FEM is appropriately biased, the output signal is 


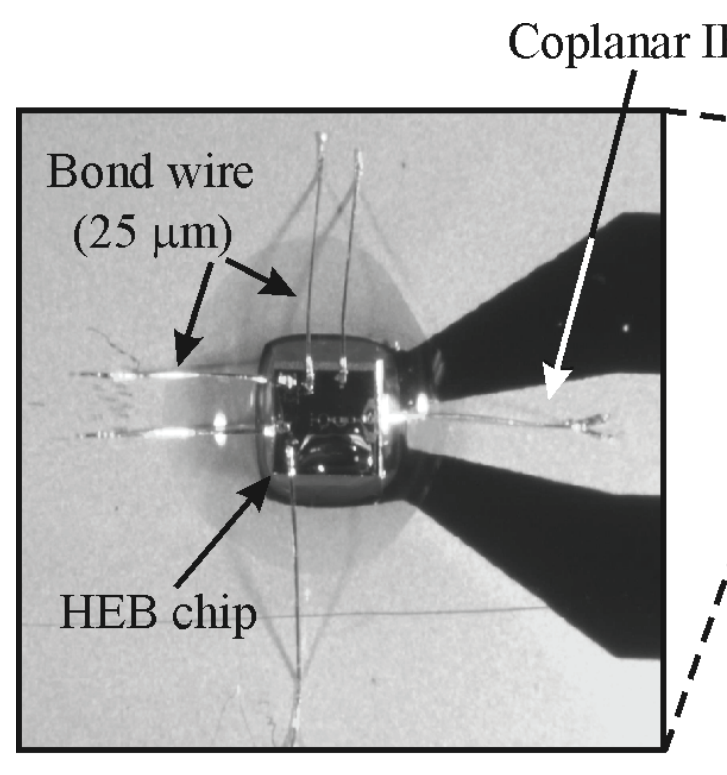

(a)

\section{feed}
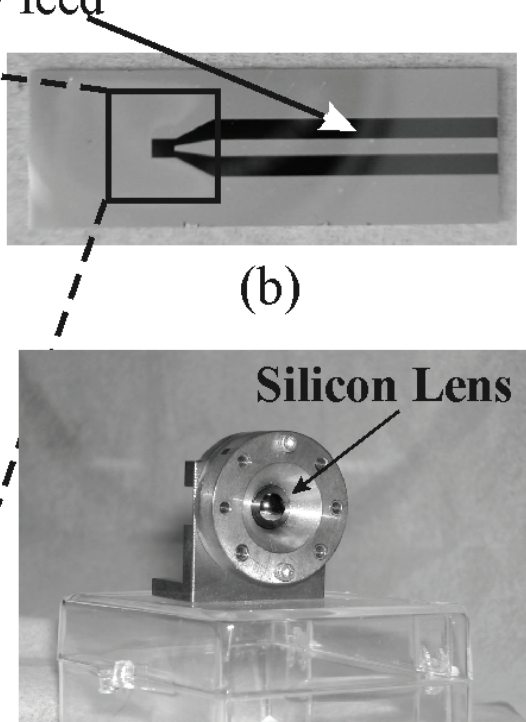

(c)

Fig. 13. Photographs of (a) the bolometer chip bonded in the quasi-optical system, (b) the coplanar IF feed for the antenna (on silicon), and (c) the cryostat lens mount. The chip is mouted directly to the IF feed circuit in (b) with a cryogenic epoxy (figure reproduced from [35].

obtained at a frequency of 48 times that of the input signal. For example, a $12.185 \mathrm{GHz}$ signal with $16 \mathrm{dBm}$ power level is used to produce a $585 \mathrm{GHz}$ output from the FEM. According to the specifications provided by VDI, the output power of the FEM varies from $0.3 \mathrm{~mW}$ to $0.9 \mathrm{~mW}$ in the range of 576 to $640 \mathrm{GHz}$, which is more than adequate for pumping the HEB devices. Both the LO and RF are coupled into the cryogenic dewar through a set of lenses, beam splitter, and mirrors. Inside the dewar, the quasi-optical mixer mount is placed in front of the Teflon window and biased using a bias-T while the IF signal is output through an isolator and low noise amplifier (LNA) before being fed to the external IF chain.

With this measurement setup, the HEB pumped I-V curves at $585 \mathrm{GHz}$ were initially measured by coupling only LO power into the dewar and to the device. As shown in Fig. 15, the un-pumped critical current for the HEB under test is $110 \mu \mathrm{A}$, close to the value measured previously. The critical current decreases with increasing LO power, as expected, and the HEB device is fully saturated (behaves like a pure resistance) at an LO power of P4, indicating that the entire $\mathrm{Nb}$ microbridge is pumped from the superconducting state into the normal state [35]. Note that the actual power received by the mixer is not measured directly, although it can (in principle) be estimated from the losses predicted in the system optics and RF circuitry. Nevertheless, The I-V curves measured demonstrate that adequate power is available for pumping the mixer into saturation. The measured E-plane radiation pattern of the lens-coupled annular-slot antenna shown in Fig. 15(b) demonstrates good agreement with calculation. 


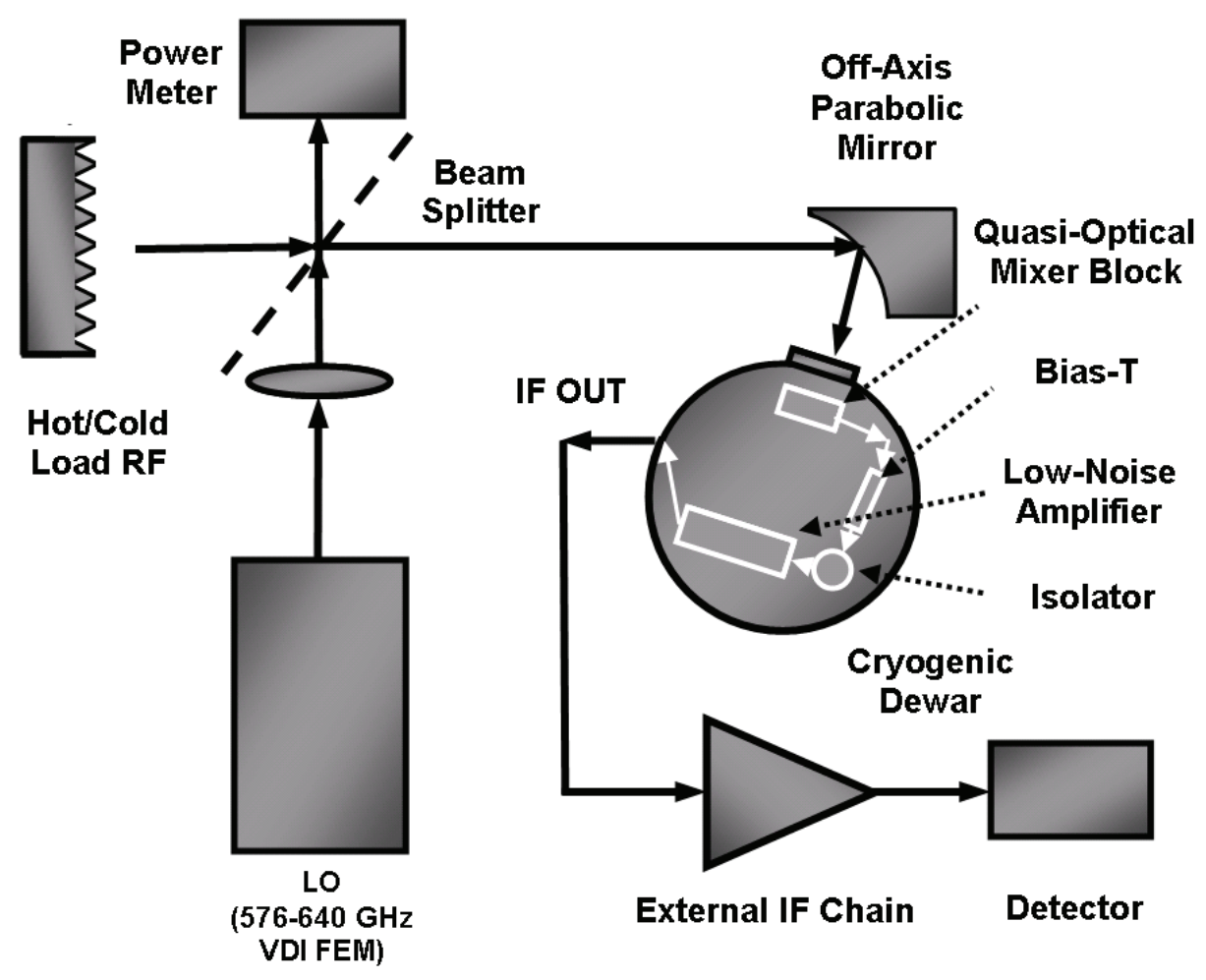

Fig. 14. RF and Y-factor measurement setup for characterizing the HEB mixer performance including gain and noise temperature.

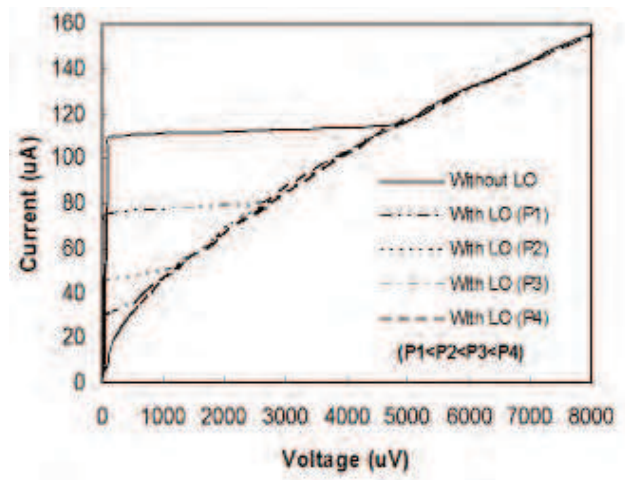

(a)

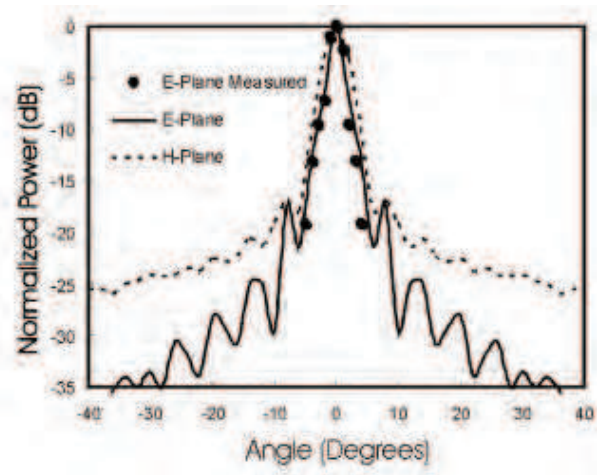

(b)

Fig. 15. (a) Pumped and un-pumped I-V curves measured at $585 \mathrm{GHz}$ with a LO power provided by a VDI 576-640 GHz FEM. (b) Measured direct response vs. incident angles at 585 $\mathrm{GHz}$ compared to calculated antenna radiation E-Plane radiation pattern (H-plane pattern is also plotted using dotted line). 

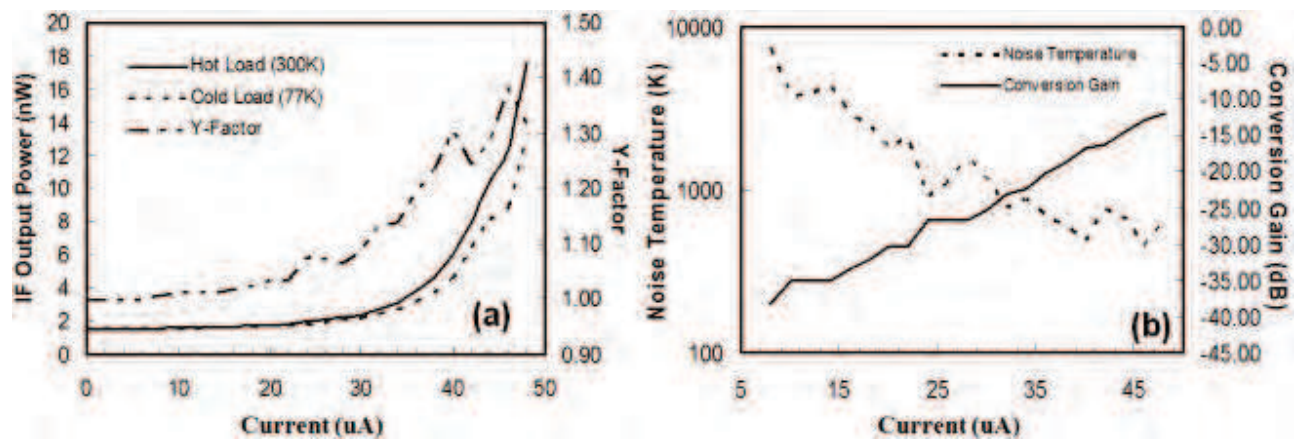

Fig. 16. (a) Measured IF output power and Y-factor as functions of current, and (b) corrected mixer conversion gain and SSB noise temperature vs. current.

Y-factor measurement was performed to characterize the HEB mixer gain and noise temperature. Figure 3.12 (a) shows the IF output power (at $1.8 \mathrm{GHz}$ ) together with the corresponding Y-factor at $4.3 \mathrm{~K}$ as functions of bias current in the case of a hot (300K) and cold (77K) load. It can be seen that the highest IF output power is measured at bias currents just before the device is fully saturated. Above this biasing point, the d-HEB becomes saturated and acts as a normal resistor. The highest Y-factor measured is approximately $1.20 \mathrm{~dB}$, corresponding to an uncorrected DSB receiver noise temperature of $T_{\text {rec,DSB }} \sim 650 \mathrm{~K}$ [35].

To calculate the mixer gain and noise temperature, the noise contribution from the RF optics and the IF chain must be removed from the measured data. Table 3.1 lists the gain and noise temperature contribution for the optical components. The total loss from the RF optics is estimated to be $G_{r f}=-1.73 \mathrm{~dB}$ and the equivalent Planck noise temperature is thus $T_{r f}=114.6$ $\mathrm{K}$. The cryogenic LNA has a gain of $G_{i f}=42 \mathrm{~dB}$ and a noise temperature of $T_{i f}=3.0 \mathrm{~K}$ at 1.8 $\mathrm{GHz}$. After correction, the effective noise level for the hot/cold $(300 \mathrm{~K} / 77 \mathrm{~K})$ load at the input of the mixer is then $278.6 \mathrm{~K} / 128.8 \mathrm{~K}\left(T_{\text {eff,hot }} / T_{\text {eff,cold }}\right)$. The mixer conversion gain is calculated by:

$$
G_{\text {mixer }}=\frac{\Delta P_{\text {out }}}{\Delta P_{\text {in }} G_{i f}}=\frac{\Delta P_{\text {out }}}{2 k B\left(T_{\text {eff, hot }}-T_{\text {eff }, \text { cold }}\right) G_{i f}}
$$

where $\Delta P_{\text {in }}$ and $\Delta P_{\text {out }}$ are input and output power change, $\mathrm{k}$ is Boltzmann constant, $\mathrm{B}$ is the IF bandwidth ( $\mathrm{B}=1.0 \mathrm{GHz}$ for estimation since the external IF chain is not used for this measurement). The mixer DSB noise temperature can be found from,

$$
T_{\text {receiver }}=T_{r f}+\frac{T_{\text {mixer, } D S B}}{G_{i f}}+\frac{T_{i f}}{G_{i f} G_{\text {mixer }}}
$$

according to equation 3.5, and the SSB mixer noise temperature is then $T_{\text {mixer, } S S B}=$ $2 T_{\text {mixer, } D S B}$. The mixer gain and SSB noise temperature are shown in Figure $3.12(\mathrm{~b})$ as functions of bias current. Biased at 48 ţA and a bath temperature of $4.3 \mathrm{~K}$, the mixer gain at $585 \mathrm{GHz}$ is $-11.9 \mathrm{~dB}$ and the SSB mixer noise temperature is $T_{m i x, S S B} \sim 630 \mathrm{~K}$ [35].

\subsection{THz HEB mixer focal plane arrays for imaging applications}

Highly-sensitive receivers employing superconducting hot-electron bolometers (HEB's) have been intensively studied and applied in millimeter-wave and far-infrared (FIR) imaging and 
remote sensing in recent years [1], [11]. However, in many applications, only one pixel of object information from receiver is insufficient. To effectively map the spatial distribution of radiation intensity, many pixels of imaging information are usually needed. Although mechanical scanning can be applied to a single element mixer to fulfill the above requirement, in some cases, this is not feasible due to the long observing time required to form a complete image [36], [37]. For example, in applications such as plasma diagnostics, information is often needed on a time scale of a microsecond, which is not usually possible with a single element detection system with mechanical scanning [18]. Imaging mixer arrays [13, 18] are the best approach for these applications since they can greatly reduce observing and processing time by recording imaging information in parallel.

Imaging arrays for submillimeter applications are a subject of continuing interest for both the astronomy and chemical spectroscopy communities. A number of researchers have put considerable effort into developing imaging arrays based on Schottky diodes or superconducting detectors [21, 39]. In 1982, D. B. Rutledge and Muha proposed a high-resolution imaging antenna array diagram with a "reverse-microscope" optical configuration [13]. On the bases of this diagram, a research group at the University of California at Davis is currently working on a $90 \mathrm{GHz}$ Schottky diode mixer array with bow-tie antennas [21]. Bow-tie antennas, however, have a number of drawbacks for high resolution imaging applications in the $\mathrm{THz}$ region since they are not compact for single imaging element design and exhibit antenna patterns with maximum off the antenna bore-sight [20]. JPL has proposed a 1.6 THz 1-D array based on diagonal horns antennas [39]. However, 2-D arrays based on this scheme are difficult to realize. In recent years, another approach called the "fly's-eye concept" has been investigated at the University of Massachusetts at Amherst [38]. A 3-element HEB focal plane array based on this concept has also been reported recently demonstrating promising performance [38]. For this approach, each mixer element uses a separate imaging lens, which presents difficulties for design and fabrication, and hence the imaging resolution of this system will be limited.

Fig. 17 shows a diagram of HEB imaging array on an extended hemispherical silicon lens investigated in this work. Each of the imaging elements employs an annular-slot antenna as the coupling component into which a HEB device is integrated. Two difficulties have to be addressed to achieve a high-resolution imaging array. First, from the Shannon-Whittaker condition [40], the array element spacing should not exceed one wavelength at the frequency of interest for diffraction-limited imaging. Thus, very limited room is left for accommodating those circuits such as low-pass filters (LPF) and low-noise amplifiers (LNA). Second, while maintaining a small array element spacing is required to achieve high imaging resolution, mutual coupling between adjacent annular slot antennas and the cross-talk between the IF outputs become important and can limit increased resolution by simple scaling of the array geometry.

To achieve a diffraction-limited imagine with an HEB mixer array at submillimeter frequencies, the "reverse-microscope" optical configuration proposed by D. B. Rutledge and Muha [13] is utilized. Figure 18 shows a diagram of this configuration in which the annular slot antenna array substrate is attached to the back side of the imaging lens and an objective lens is placed in front of the imaging lens. The image signal is focused through both lenses onto the mixer array. The image of the object is then reconstructed by plotting the IF output signal from each element in the array. As discussed in previous sections, by utilizing the same material (silicon) for both the array substrate and the imaging lens in this configuration, the 


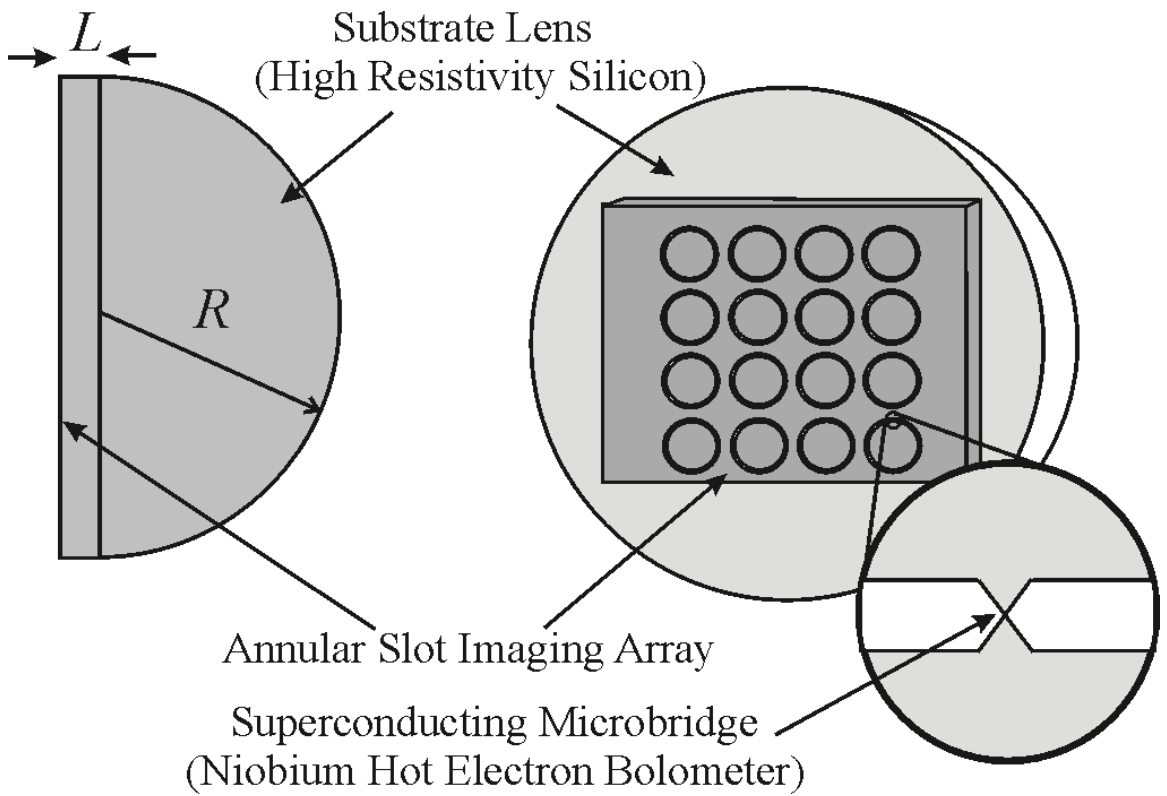

Fig. 17. Schematic of a HEB mixer imaging array using annular-slot antenna mounted on an extended hemispherical silicon lens with radius $\mathrm{R}$ (extension length $\mathrm{L}$ ).

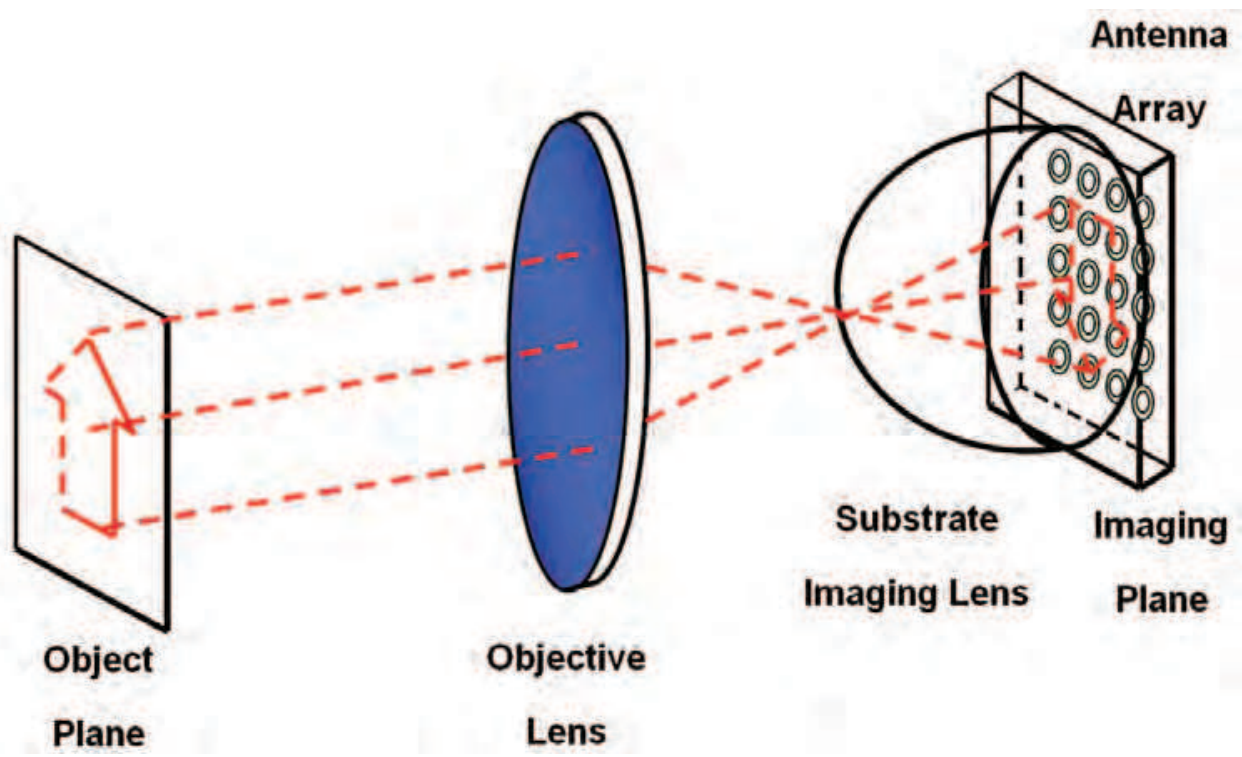

Fig. 18. Diagram of the reverse-microscope configuration proposed by D. B. Rutledge [13]. This configuration provides a capability of diffraction-limited imaging at $\mathrm{THz}$ frequencies. 

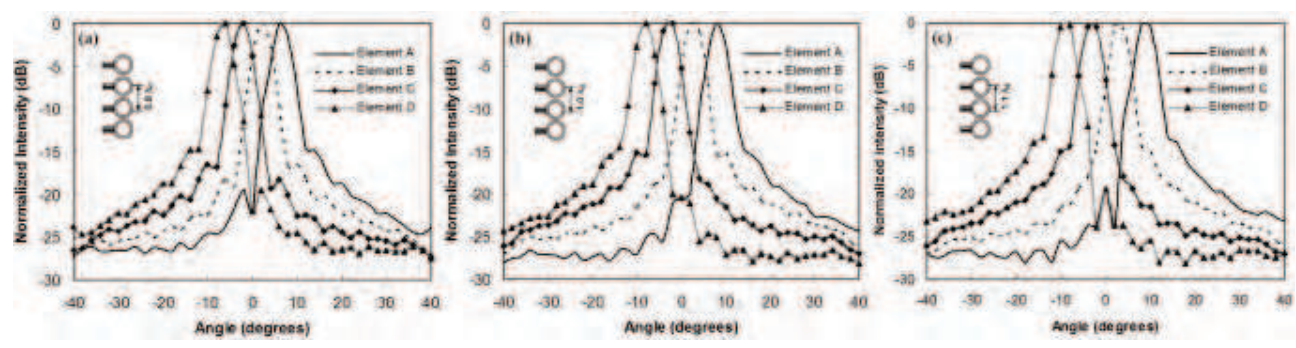

Fig. 19. Off-axis element radiation patterns for the lens-coupled one-D annular-slot antenna arrays calculated using ray-tracing technique. Patterns are calculated with element spacings of (a) $0.8 \lambda_{d}$, (b) $1.0 \lambda_{d}$, and (c) $1.1 \lambda_{d}$.

trapped surface-wave is eliminated and the cross-talk between adjacent imaging elements is reduced, allowing a high imaging resolution to be achieved [13].

The single element HEB mixers employing annular slot antennas developed and fabricated in the earlier work in this research have shown promising performance. Here, the basic mixer design including the antenna, impedance matching circuits and low-pass filters are applied and expanded to realize a full imaging mixer focal-plane array. Due to the small element spacing $\left(\sim \lambda_{d} \approx 150 \mu \mathrm{m}\right)$ for a diffraction-limited imaging, the mutual coupling between adjacent annular slot antennas in the mixer array is investigated to check whether it needs to be included in the design considerations. Both ADS Momentum simulations and EMF analysis [41,42] were performed to study the mutual-impedances of the annular-slot antenna array with various element spacing, and a conclusion has been drawn that when the element spacing is larger than $0.8 \lambda_{d}$ (while smaller than $1.0 \lambda_{d}$ ), the antenna mutual impedances and hene the cross talk between the adjacent elements are small enough that their effect can be safely neglected in the design of a diffraction-limited imaging system.

The element annular slot antenna (lens-coupled) off-axis radiation patterns for various spacings in prototype one-D $(1 \times 4)$ and two-D $(2 \times 2)$ arrays have been calculated using the ray-tracing technique. As shown in Fig. 19, the element antenna pattern in the one-D arrays has a 3-dB beam width of $\theta_{3-d B} \sim 4^{\circ}$ with side-lobe levels less than $-10 \mathrm{~dB}$. The beam spacings between adjacent antennas are $\Delta \theta \sim 4.0^{\circ}, 5.0^{\circ}$, and $5.7^{\circ}$ with crossover power level around $-3.9 \mathrm{~dB},-6.0 \mathrm{~dB}$, and $-7.0 \mathrm{~dB}$ for $\mathrm{d}=0.8 \lambda_{d}, 1.0 \lambda_{d}$, and $1.1 \lambda_{d}$, respectively. For imaging applications, a crossover level larger than $3 \mathrm{~dB}$ is generally used as a figure of merit to distinguish between two points and quantify the spatial resolution of an array. Fig. 20 shows the annular slot antenna off-axis radiation patterns in the $2 \times 2$ arrays. The element antenna pattern in the azimuth scan has the same properties as that in the one- $\mathrm{D}$ array. The element antenna pattern in the elevation scan has a 3-dB beam width of $\theta_{3-d B} \sim 3.5^{\circ}$ with side-lobe levels less than $-10 \mathrm{~dB}$. The beam spacings between adjacent antennas are $\Delta \theta \sim 4.0^{\circ}, 5.0^{\circ}$, and $6.0^{\circ}$ with crossover power level around $-7.0 \mathrm{~dB},-11.0 \mathrm{~dB}$, and $-12.5 \mathrm{~dB}$ for $\mathrm{d}=0.8 \lambda_{d}, 1.0 \lambda_{d}$, and $1.1 \lambda_{d}$. The antenna patterns in the elevation scan (or the E-plane pattern) have a narrower 3- $\mathrm{dB}$ beam width, compared to the patterns in the azimuth scan (or the H-plane pattern).

Shown in Fig. 21 are the fabrication and assembling results of the HEB imaging mixer arrays. For typical one-D arrays in Fig. 21 (a), quarter-wavelength impedance transformers were utilized. While twin-HEB devices were integrated in the two-D arrays due to limited spacing between adjacent antennas. The twin-HEB device has two HEB microbridges fabricated in 

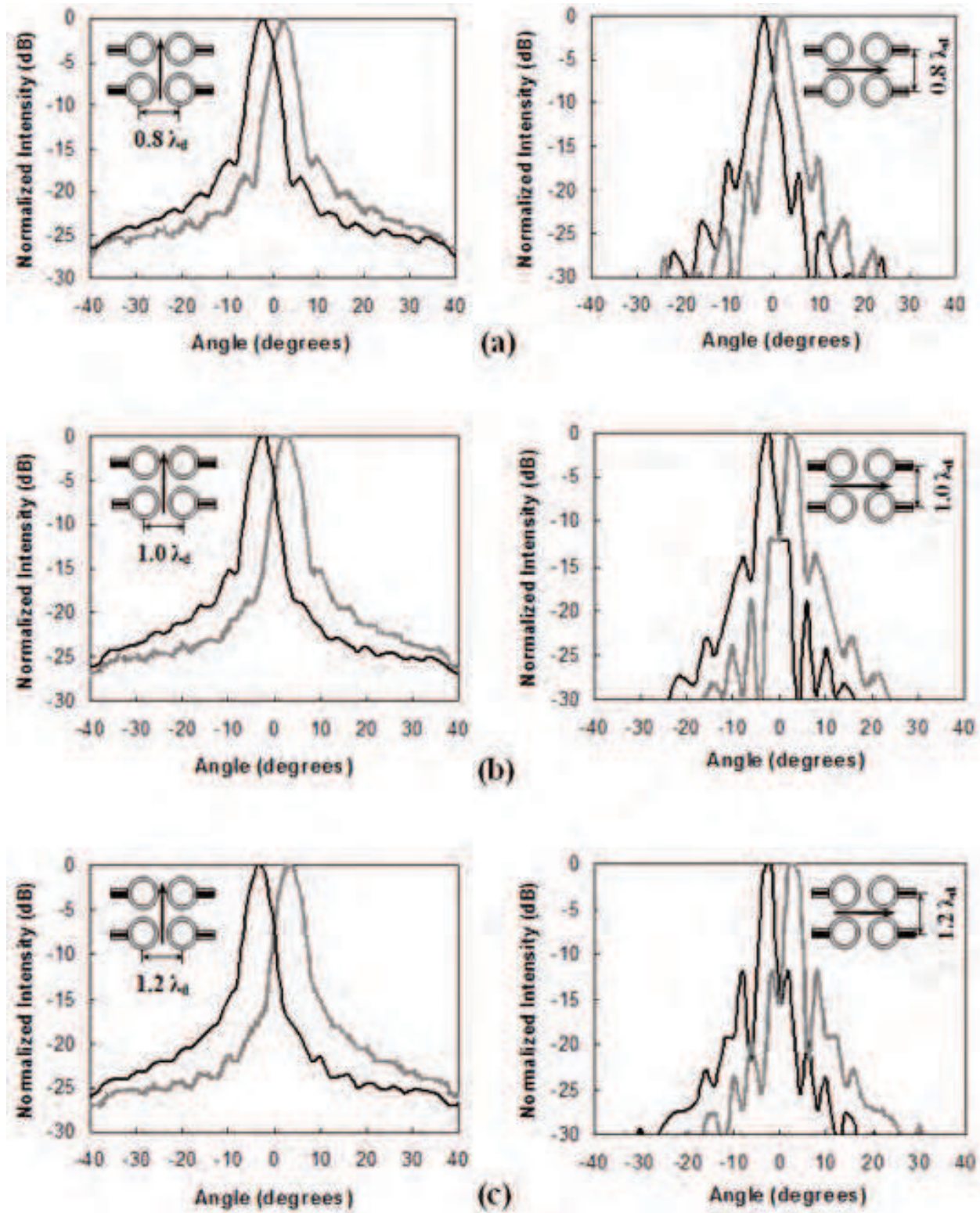

Fig. 20. Off-axis element radiation patterns in the elevation scan and azimuth scan for the lens-coupled two-D annular-slot antenna arrays calculated using ray-tracing technique. Patterns are calculated with element spacings of (a) $0.8 \lambda_{d}$, (b) $1.0 \lambda_{d}$, and (c) $1.2 \lambda_{d}$. 

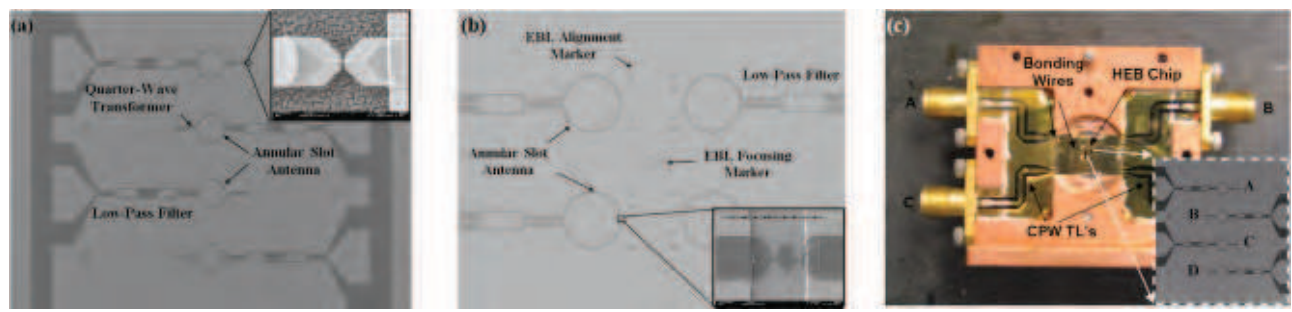

Fig. 21. Lens-antenna coupled HEB mixer imaging array fabrication and assembling results:

(a) SEM picture of an one-D HEB imaging array with quarter-wave impedance transformer,

b) SEM picture of a two-D HEB imaging array using twin-HEB devices, and (c) the assembled mixer array mount, ready for cryogenic testing.

series (each with a normal-state resistance of $50 \Omega$ ) and connected by a gold cooling pad in bewteen, offering a device impedance of $100 \Omega$ (matching to the antenna imbedding impedance). This approach eliminates the need for a lossy transmission line but requires a slightly more complex fabrication process. To measure the array performance, a quasi-optical mixer array block has been fabricated. As shown in Fig. 21 (c), the HEB mixer array chip was first bonded to a silicon substrate ( $\sim 1.1 \mathrm{~mm}$ thick with four bended CPW transmission lines) using cryogenic epoxy, and an ultrasonice wire-bonding tool is used t oelectrically connect both the grounds and center conductors. Two other silicon substrates with CPW trsnsmission lines have been installed into the backside of the array block, and four subminiature A (SMA) connectors are utilized to output the IF signals.

A close-cycled cryocooler has been employed in the mixer array RF measurements. Inside the cryocooler as shown in Fig. 22, a $585 \mathrm{GHz}$ mesh-filter ( $\sim 40 \mathrm{GHz}$ bandwidth) is placed on the $40 \mathrm{~K}$ stage just in front of the Teflon window. The assembled mixer array block is installed with four outputs connected to the four bias-T's. The four-way Miteq power combiner, Durado cryogenic isolator and Miteq low-noise amplifier are anchored to the $4.2 \mathrm{~K}$ stage with specially designed copper mounting pieces for good thermal contact. The RF components are connected using semi-rigid coaxial cables. An IF feedthrough is designed to couple the microwave signals out of the cryocooler. For better thermal insulation, the inside coaxial cable is coiled and the cable jacket is soldered to the copper heat sink, which is anchored to the $40 \mathrm{~K}$ stage. After assembling all the inside components, the unused windows of the cover plate are covered with aluminum foils for further blocking the room-temperature radiation. During the HEB mixer array operation, only one of the four HEB devices is dc-biased at one time, and thus, each mixer can be measured separately.

Prior to RF testing, the DC charactersitcs of an one-D array with four mixer elements (A-D) were measured. A sharp transition from the superconducting state to the normal state is observed at around $5.5 \mathrm{~K}$ for elements $\mathrm{A}, \mathrm{B}$ and $\mathrm{C}$ with $\Delta T_{C} \sim 0.5 \mathrm{~K}$. The array element $\mathrm{D}$ became an open circuit at a temperature of $\sim 190 \mathrm{~K}$ because the bond wire lost contact to the center conductor of the CPW transmission line due to the mechanical vibration from the close-cycled cryocooller system. The current-voltage (I-V) curves are then measured at a bath temperature of $\sim 3.9 \mathrm{~K}$, as shown in Fig. 22 (b). The critical currents were measured to be 290 $\mu A, 300 \mu A$ and $400 \mu A$ for element $\mathrm{A}, \mathrm{B}$, and $\mathrm{C}$, respectively.

To measure the HEB mixer FPA imaging angular resolution, two adjacent elements (element $B$ and $C$ with $B$ in the upper position) in an one dimensional array chip (see inset of Fig. 21(c), 
element spacing $d=1.0 \lambda_{d}$ ) were DC biased and the responses were monitored. During the experiment, the VDI $585 \mathrm{GHz}$ solid state source is employed and the distance between the silicon lens and the source is nearly $50 \mathrm{~mm}$ for maximum response. The responses for element $\mathrm{B}$ and $\mathrm{C}$ are normalized and plotted in Fig. 22(c) together with the off-axis antenna patterns (see Fig. 19) predicted using the ray-tracing technique. Again, reasonable agreement has been obtained. The discrepancies can be attributed to errors in source position measurement and the nonlinear relationship between the current response and the absorbed RF power. The vertical distance between the two maximum response position is $4.4 \mathrm{~mm}$, resulting in an angular resolution of $5.04^{\circ}$, which is very close to the theoretical prediction (see Fig. 19).

By using an optical lens as an objective lens, the system imaging resolution can be further improved. Experiments have been performed with a distance of $133.1 \mathrm{~mm}$ between the silicon lens and the $585 \mathrm{GHz}$ solid-state source. The optical lens is placed $49.3 \mathrm{~mm}$ from the cryocooler window ( $86.3 \mathrm{~mm}$ from the silicon lens). The response peaks from the element $\mathrm{B}$ and $\mathrm{C}$ are measured at source displacement of $69.35 \mathrm{~mm}$ and $72.10 \mathrm{~mm}$, corresponding to an imaging resolution of $\sim 2.75 \mathrm{~mm}$. Compared to the results reported by the University of Massachusetts group (38) at $1.6 \mathrm{THz}$ with a three-element HEB array based on the "fly's-eye" concept [38], the integrated HEB mixer arrays on the basis of the "reverse-microscope" configuration provide a capability to realize diffraction-limited, high-resolution focal-plane arrays in the $\mathrm{THz}$ frequency region.

Y-factor measurements have been performed to each of the two array mixer elements (element $\mathrm{B}$ and $\mathrm{C}$ ) and the results show that DSB mixer noise temperatures of $1675 \mathrm{~K}$ and $3517 \mathrm{~K}$, with mixer conversion gain of $-14.73 \mathrm{~dB}$ and $-17.74 \mathrm{~dB}$, respectively, have been obtained for the two adjacent elements in the one dimensional focal-plane mixer array, which is comparable to the results reported in the literature. To the author's best knowledge, the HEB mixer focal plane array described in this paper is the first heterodyne FPA reported on the basis of the "reverse-microscope" architecture with the capability of diffraction-limited imaging. With this architecture, all the array antennas, HEB devices and IF circuits are integrated onto one silicon wafer, thus providing an alternative way for developing large FPA's in the THz region.

The measurement results in Section IV (B) are comparable to that reported in the literature [38]. However, the measured noise temperatures for elements in an array are not as good as that for a single element HEB mixer presented in Section IV (A) in this paper. Although further work needs to be done to fully understand this discrepancy, we attribute this to several possible
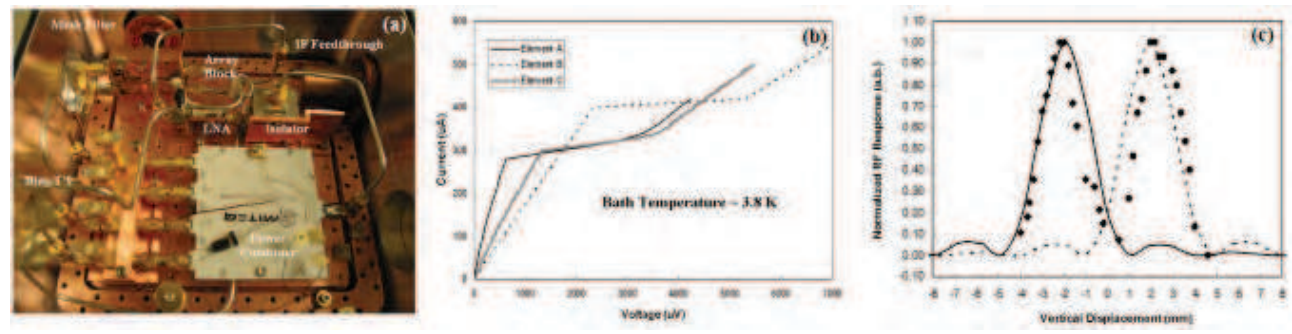

Fig. 22. Lens-antenna coupled HEB mixer imaging array characterization and results: (a) a photograph showing the components inside the close-cycled cryocooler, (b) I-V curves for HEB devices A, B and C in a one-D array, and (c) measured normalized response (dots) compared to the theoretical results by ray-tracing technique (solid- and dotted- lines). 
reasons: (1) the annular slot antennas are off-axis positioned at the back side of the silicon lens and this could introduce as large as $10 \mathrm{~dB}$ coupling loss; (2) the array receiver system used in this research is not optimized. A four-way power combiner is utilized introducing $\sim 6$ - $\mathrm{dB}$ loss, which further increases the receiver noise level; (3) the array assembly strategy is designed for prototype demonstration. Wire-bonding technique is used for electrical connection, which unavoidably deteriorates the IF performance of the mixer array circuits.

\section{Conclusion}

In this chapter, the basic theory and mechanism for lens-antenna coupled HEB THz mixers have been introduced followed by our work on $585 \mathrm{GHz}$ HEB mixers and mixer arrays based on lens-coupled ASAs. In conclusion, we have designed and fabricated hot-electron bolometer mixers, for $\mathrm{THz}$ heterodyne detection and imaging. The HEBs are integrated into lens-coupled annular-slot antennas that incorporate low-pass filters. DC and RF characterizations have been performed and a mixer conversion gain of $-11.9 \mathrm{~dB}$ and a DSB receiver noise temperature of $\sim 650 \mathrm{~K}$ have been achieved. On the basis of the single element mixer design, prototype mixer focal-plane arrays comprised of ASAs integrated with hot-electron bolometers have been developed for imaging applications at $585 \mathrm{GHz}$. Both EMF analysis and ADS momentum simulations have been performed to study the self- and mutual- impedances of the annular slot array with various element spacings. The element antenna off-axis radiation patterns have been calculated using the ray-tracing technique and the imaging angular resolution has been predicted. Initial imaging experiment results are presented and show excellent agreement with theory and simulation data, demonstrating that a diffraction-limited imaging with a resolution of $\sim 2.75 \mathrm{~mm}$ has been achieved at $585 \mathrm{GHz}$. Y-factor measurements show that DSB mixer noise temperatures of $1675 \mathrm{~K}$ and $3517 \mathrm{~K}$, with mixer conversion gain of -14.73 $\mathrm{dB}$ and $-17.74 \mathrm{~dB}$, respectively, have been obtained for two adjacent elements in a one-D focal-plane mixer array. The lens-antenna coupled HEB mixer focal plane array described in this chapter provides an promising architecture for developing focal-plane arrays that capable of diffraction-limited imaging in the $\mathrm{THz}$ region.

\section{References}

[1] D. W. Floet, "Hotspot Mixing in THz Nibium Superconducting Hot Electron Bolometer Mixers," Ph.D. Dissertation, Delft University of Technology, September 2001.

[2] R. B. Bass, "Hot-electron bolometers on ultra-thin silicon chips with beam leads for a 585 GHz receiver," Proceedings of the IEEE, vol. 80, no. 11, pp. 1662-1678, November 1992.

[3] C. E. Groppi, and J. H. Kawamura, "Coherent detector arrays for terahertz astrophysics applications," IEEE Trans. Terahertz Sci. Tech., vol. 1, no. 1, pp. 85-96, September, 2011.

[4] C. Kulesa, "Terahertz spectroscopy for astronomy: from comets to cosmology," IEEE Trans. Terahertz Sci. Tech., vol. 1, no. 1, pp. 232-240, September, 2011.

[5] J. W. Waters, "Submillimeter-wavelength heterodyne spectroscopy and remote sensing of the upper atmosphere," Proceedings of the IEEE, vol. 80, no. 11, pp. 1679-1701, 1992.

[6] M. Chipperfield, "Satellite maps ozone destroyer," Nature, vol. 362, no. 15, pp. 592, December 1988.

[7] N. C. Luhmann, Jr., "Instrumentation and techniques for plasma diagnostics: An overview," Infrared and Millimeter Waves, vol. 2, , pp. 1-65, K. J. Button, Ed. New York: Academic, 1979. 
[8] J. M. Schuchardt, J. M. Newton, T. P. Morton, and J. A. Gagliano, "The coming of MM-wave forward looking imaging radiometers," Microwave J., pp. 45-62, 1981.

[9] K. Ajito and Y. Ueno, "THz chemical imaging for biological applications," IEEE Trans. Terahertz Sci. Tech., vol. 1, no. 1, pp. 293-300, September, 2011.

[10] T. R. Globus, M. L. Norton, M. I. Lvovska, D. A. Gregg, T. B. Khromova, and B. L. Gelmont, "Reliability analysis of $\mathrm{THz}$ characterization of modified and unmodified vector sequences," IEEE Sensors Journal, vol. 10, no. 3, March 2010.

[11] E. Brown. D. Woolard, A. Samuels, T. Globus and B. Gelmont, "Remote detection of bioparticles in the THz region," IEEE MTT-S Int. Microwave Symp. Digest, no. 3, pp. 1591-1594, 2002.

[12] D. Mittleman, M. Gupta, R. Neelamani, R. Baraniuk, J. Rudd, and M. Koch, “Recent advances in terahertz imaging," Appl. Physics B, vol. 68, pp. 1085-1094, 1999.

[13] D. B. Rutledge, and Michael S. Muha, "Imaging Antenna Arrays," IEEE Trans. Antennas and Propagation, vol. AP-30, no. 4, July 1982.

[14] K. B. Cooper, R. J. Dengler, N. Llombart, B. Thomas, G. Chattopadhyay, and P. H. Siegel, "THz imaging radar fro standoff personnel screening," IEEE Trans. Terahertz Sci. Tech., vol. 1, no. 1, pp. 169-182, September, 2011.

[15] L. Liu, J. L. Hesler, H. Xu, A. W. Lichtenberger, and R. M. Weikle, II, “A Broadband Quasi-Optical THz Detector Using a Zero Bias Schottky Diode," IEEE Microwave and Wireless Components Letters, vol. 20, no. 9, 2010.

[16] B. S. Karasik, A. V. Sergeev, and D. E. Prober, "Nanobolometers for THz photon detection," IEEE Trans. Terahertz Sci. Tech., vol. 1, no. 1, pp. 97-111, September, 2011.

[17] D. E. Prober, "Superconducting terahertz mixer using a transition-edge microbolometer," Appl. Phys. Lett., vol. 62, pp. 2119-2121, 1993.

[18] D. P. Neikirk, "Integrated detector arrays for high resolution far-infrared imaging," Ph.D. Dissertation, California Institute of Technology, 1984.

[19] C. Smith, H. Xu, N. Barker, "Development of a multi-layer SU-8 process for terahertz frequency waveguide blocks," IEEE Microwave Theory and Technology Symposium (MTT-S) Digest, 2005.

[20] D. B. Rutledge, D. P. Neikirk, and D. P. Kasilingam, "Integrated-circuit antennas," Infrared and Millimeter Waves, vol. 10, chap. 1, Academic Press Inc., 1979.

[21] P. L. Hsu, B. H. Deng, et al, "Millimeter-wave imaging array development for microwave reflectometry and ECE imaging," Rev. Sci. Instrum., vol. 72, no. 1, 2001.

[22] D. F. Filipovic, S. S. Gearhart, and G. M. Rebeiz, "Double-slot antennas on extended hemispherical and elliptical silicon dielectric lenses," IEEE Trans. Microwave Theory Tech., vol. 41, no. 10, 1993.

[23] W. F. M. Ganzevles, L. R. Swart, J. R. Gao, P. A. J. de Korte, T. M. Klapwijk, “Direct response of twin-slot antenna-coupled hot-electron bolometer mixers designed for 2.5 THz radiation detection," App. Phys. Lett., vol. 76, no. 22, 2000.

[24] M. Hajenius, J. J. A. Baselmans, J. R. Gao, T. M. Klapwijk, P. A. J. de Korte, B. Voronov, and G. Gol'tsman, "Low nose $\mathrm{NbN}$ superconducting hot electron bolometer mixers at 1.9 and $2.5 \mathrm{THz}, "$ Supercond. Sci. Technol., vol. 17, 2004.

[25] A. D. Semenov, H. Richter, H.-W. Hubers, B. Gunther, A. Smirnov, K. S. Il'in, M. Siegel, and J. P. Karamarkovic, "Terahertz performance of integrated lens antennas with a hot-electron bolometer," IEEE Trans. Microwave Theory \& Tech., vol. 55, no. 2, 2007.

[26] B. Jackson, "Sub-mm and Far-IR Mixers Status and Outlook," SRON National Institute for Space Research, the Netherland, 2003. 
[27] B. S. Karasik and A. I. Elantiev, "Noise temperature limit of a superconducting hot-electron bolometer mixer," Appl. Phys. Lett., vol. 68, no. 6, Feburary, 1996.

[28] J. C. Schultz, A. W. Lichtenberger, J. Z. Zhang, "Nanoscale superconducting THz hot-electron bolometers fabricated with UV lithography on ultra-thin si beam-lead chips," IEEE Trans. Appl. Supercond., vol. 15, no. 2, 2005.

[29] E. Gerecht, D. Gu, X. Zhao, J. Nicholson, F. Rodriguez-Morales, and S. Yngvesson, "Development of $\mathrm{NbN}$ terahertz HEB mixers coupled through slot-ring antennas," Proc. 15th Int. Symp. Space Terahertz Tech., Amerst, 2004.

[30] N. Paravastu, and R. M. Weikle, II, “A 40-80 GHz quasi-optical balanced doubler using nested ring-slot antennas," IEEE Antenna and Propagation Symposium Diegest, 2002.

[31] C. E. Tong, and R. Blundell, "An annular slot antenna on a dielectric half-space," IEEE Trans. Antennas Propagat., vol. 42, no. 7, pp. 967-974, July 1994.

[32] S. Raman, and G. M. Rebeiz, "Single- and dual-polarized millimeter-wave slot-ring antennas," IEEE Trans. Antennas Propagat., vol. 44, no. 11, 1996.

[33] L. Liu, R. M. Weikle, II, A. W. Lichtenberger, A. Isin, B. S. Deaver, "A 585 $\mathrm{GHz}$ diffusion-cooled niobium HEB mixer element for imaging applications," 16th International Symposium on Space Terahertz Technology (ISSTT)., Geteborg, Sweden, May 2005.

[34] R. A. Wyss, B. S. Karasik, W. R. McGrath, B. Bumble and H. Leduc, "Noise and bandwidth measurement of diffusion-cooled $\mathrm{Nb}$ hot-electron bolometer mixers at frequencies above the superconductive energy gap," 10th International Symposium on Space Terahertz Technology (ISSTT)., Charlottesville, Virginia, USA, 1999.

[35] L. Liu, Q. Xiao, J. C. Schultz, H. Xu, A. W. Lichtenberger, R. M. Weikle, II, “The design, fabrication and characterization of a submillimeter-wave niobium HEB mixer imaging array based on the reverse-microscope concept," IEEE Trans. Appl. Supercond., vol. 17, no. $2,2007$.

[36] D. T. Hodges, F. B. Foote, E. E. Reber, and R. L. Schellenbaum, “Near millimeter wave radiometric imaging," Fourth Int. Conf. Infrared Millimeter Waves and Their Appl., no. 79, pp. 51-52, 1979.

[37] J. Waldman, H. R. Fetteman, P. E. Duffy, T. G. Bryant, and P. E. Tannenwald, "Submillimeter model measurements and their applications to millimeter radar systems," Fourth Int. Conf. Infrared Millimeter Waves and Their Appl., no. 79, pp. 49-50, 1979.

[38] F. Rodriguez-Morales, et. al., "A terahertz focal plane array using HEB superconducting mixers and MMIC IF amplifiers," IEEE Microwave and Wireless Components Letters, vol. 15, no. 4, pp. 199-201, Apr. 2005.

[39] G. Chattopadhyay, I. Mehdi, J. S. Ward, E. Schlecht, A. Skalare, and P. H. Siegel, "Development of multi-pixel heterodyne array instruments at submillimeter wavelength," IEEE Asia-Pacific Microwave Conference, 2004.

[40] C. E. Shannon, "Communication in the presence of noise," Proceedings of the IRE, vol. 37, pp. 10-21, 1949.

[41] L. Liu, Q. Xiao, A. W. Lichtenberger, and R. M. Weikle, II, "Integrated $585 \mathrm{GHz}$ hot-electron mixers based on annular slot antennas," The 2007 International Microwave Symposium, Howaii, USA, Jun, 2007.

[42] L. Liu, H. Xu, A. W. Lichtenberger, and R. M. Weikle, II, “Integrated $585 \mathrm{GHz}$ Hot-Electron Mixer Focal-Plane Arrays Based on Annular-Slot Antennas," IEEE Trans. Microwave Theory Tech., vol. 58, no. 7, 2010. 


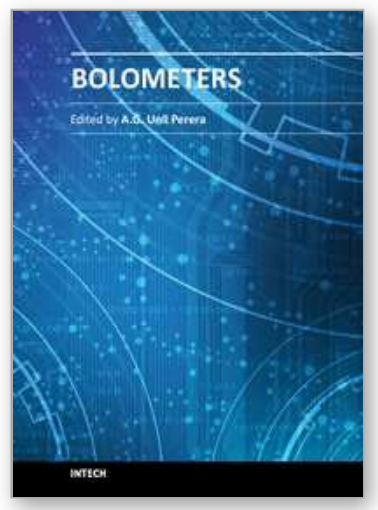

\author{
Bolometers \\ Edited by Prof. Unil Perera
}

ISBN 978-953-51-0235-9

Hard cover, 196 pages

Publisher InTech

Published online 09, March, 2012

Published in print edition March, 2012

Infrared Detectors and technologies are very important for a wide range of applications, not only for Military but also for various civilian applications. Comparatively fast bolometers can provide large quantities of low cost devices opening up a new era in infrared technologies. This book deals with various aspects of bolometer developments. It covers bolometer material aspects, different types of bolometers, performance limitations, applications and future trends. The chapters in this book will be useful for senior researchers as well as beginning graduate students.

\title{
How to reference
}

In order to correctly reference this scholarly work, feel free to copy and paste the following:

Lei Liu (2012). Lens-Antenna Coupled Superconducting Hot-Electron Bolometers for Terahertz Heterodyne Detection and Imaging, Bolometers, Prof. Unil Perera (Ed.), ISBN: 978-953-51-0235-9, InTech, Available from: http://www.intechopen.com/books/bolometers/lens-antenna-coupled-hot-electron-bolometers-for-terahertzheterodyne-detection-and-imaging

\section{INTECH}

open science / open minds

\section{InTech Europe}

University Campus STeP Ri

Slavka Krautzeka 83/A

51000 Rijeka, Croatia

Phone: +385 (51) 770447

Fax: +385 (51) 686166

www.intechopen.com

\section{InTech China}

Unit 405, Office Block, Hotel Equatorial Shanghai

No.65, Yan An Road (West), Shanghai, 200040, China

中国上海市延安西路65号上海国际贵都大饭店办公楼405单元

Phone: +86-21-62489820

Fax: +86-21-62489821 
(C) 2012 The Author(s). Licensee IntechOpen. This is an open access article distributed under the terms of the Creative Commons Attribution 3.0 License, which permits unrestricted use, distribution, and reproduction in any medium, provided the original work is properly cited. 